\title{
Efficacy and side effects of intravenous theophylline in acute asthma: a systematic review and meta-analysis
}

This article was published in the following Dove Press journal:

Drug Design, Development and Therapy

\author{
Gulixian Mahemuti \\ Hui Zhang \\ Jing $\mathrm{Li}$ \\ Nueramina Tieliwaerdi \\ Lili Ren
}

Respiratory Department, The Second Affiliated Hospital of Xinjiang Medical University, Urumqi, Xinjiang Province, People's Republic of China
Correspondence: Gulixian Mahemuti Respiratory Department, The Second Affiliated Hospital of Xinjiang Medical University, No 38 Nanhu East Road, Urumqi 830063, Xinjiang Province, People's Republic of China Email gulixianmahemuti@I26.com
Background and objective: Theophylline has been used for decades to treat both acute and chronic asthma. Despite its longevity in the practitioner's formulary, no detailed meta-analysis has been performed to determine the conditions, including concomitant medications, under which theophylline should be used for acute exacerbations of asthma. We aimed to quantify the usefulness and side effects of theophylline with or without ethylene diamine (aminophylline) in acute asthma, with particular emphasis on patient subgroups, such as children, adults, and concomitant medications.

Methods: We searched PubMed, EMBASE, The Cochrane Library, ClinicalTrials.gov, and the WHO Clinical Trials Registry for randomized, controlled clinical trials. We planned a priori subgroup analyses by time post-medication, concomitant medication, control type, and age.

Results: We included 52 study arms from 42 individual trials. Of these, 29 study arms included an active control, such as adrenaline, beta-2 agonists, or leukotriene receptor antagonists, and 23 study arms compared theophylline (with or without ethylene diamine) with placebo or no drug. Theophylline significantly reduced heart rate when compared with active control $(p=0.01)$ and overall duration of stay ( $p=0.002$ ), but beta- 2 agonists were superior to theophylline at improving forced expiratory volume in one second (FEV1) $(p=0.002)$. Theophylline was not significantly different from other drugs in its effects on respiratory rate, forced vital capacity (FVC), peak expiratory flow rate, admission rate, use of rescue medication, oxygen saturation, or symptom score. Closer examination of the data revealed that the medications given in addition to theophylline or control significantly changed the effectiveness of theophylline (subgroup difference: $p<0.00001$ ).

Conclusion: Given the low cost of theophylline, and its similar efficacy and rate of side effects compared with other drugs, we suggest that theophylline, when given with bronchodilators with or without steroids, is a cost-effective and safe choice for acute asthma exacerbations.

Keywords: theophylline, theophylline with ethylene diamine, aminophylline, asthma, bronchodilators, beta-2 agonists, adrenaline, FEV, PEFR, affordable drugs

\section{Introduction}

Acute asthma exacerbations are a frequent and serious reason for presentation to hospital emergency departments. Asthma prevalence in adults globally is estimated at $4.3 \%$, with Australia, the UK, Sweden, and the Netherlands all exceeding $15 \% .{ }^{1}$ In children, the prevalence is even higher, with many countries reporting asthma rates in children over $20 \%{ }^{2}$ In many parts of the world, asthma prevalence in increasing, although in some countries with high rates of asthma, the prevalence may now be levelling off. ${ }^{3}$

Severe asthma exacerbations in children or adults are very serious and can be lifethreatening. According to the World Health Organization, asthma causes $\sim 250,000$ 
deaths worldwide each year. ${ }^{4}$ Despite a range of drugs for the treatment of asthma, ${ }^{5}$ systematic evidence for the efficacy of these drugs is not universal. Thus, especially in developing countries, it is essential that the comparative effectiveness of all asthma treatments, including older and more affordable drugs, be available to health practitioners.

Theophylline, a methylxanthine, is a bronchodilator. When combined with ethylene diamine as "aminophylline", it is more soluble and is thus the more common form of theophylline used for intravenous (IV) administration. ${ }^{6,7}$ Available in generic form, theophylline with or without ethylene diamine is certainly affordable. However, its efficacy, especially in children, and the effective doses are a matter of dispute. We therefore undertook this study to compare the effectiveness of IV theophylline with all available comparators.

\section{Methods}

The current systematic review and meta-analysis was performed on the principles of the Cochrane Collaboration. ${ }^{8}$

\section{Data sources and search strategy}

We searched PubMed, EMBASE, the Cochrane Library, ClinicalTrials.gov, and the WHO international clinical trials registry for relevant articles. Our search strategy used the following keywords, as full-text and MESH terms (where appropriate): (Theophylline OR 1,3-dimethylxanthine OR Elixophyllin OR Norphyl OR Phyllocontin OR QuibronTSR OR Theo-24 OR TheoCap OR Theochron OR TheoDur OR Theo-Time OR Truxophyllin OR Uniphyl OR aminophylline) AND ("Short-acting beta2 agonist" OR "short-acting beta agonist" OR "beta* adrenergic receptor agonist" OR SABA OR salbutamol OR formoterol OR eformoterol OR "long-acting beta agonist" OR LABA OR albuterol OR levalbuterol OR betamethasone OR hydrocortisone OR methylprednisolone OR prednisolone OR Ventolin OR Proventil OR Atock OR Atimos OR Foradil OR Oxis OR Perforomist OR salmeterol OR bambuterol OR fluticasone OR budesonide OR glucocorticoid OR Flixotide OR Flixonase OR Pulmicort OR Rhinocort OR anticholinergic OR ipratropium OR epinephrine OR beclamethasone OR montelukast OR zafirlukast OR "5-LOX inhibitor" OR cromolyn OR placebo OR no drug) AND Asthma AND (Intravenous OR IV OR iv) AND (RCT OR random OR randomised OR randomized OR groups OR "randomised controlled trial" OR "randomized controlled trial” OR "controlled clinical trial"). No date or language restrictions were applied. All citations were uploaded into EPPI-Reviewer $4^{9}$ and were independently coded by two investigators. The date of the last search was 9 July 2017.

\section{Inclusion criteria}

Citations were included if they matched the following PICOTS: the population was children or adults presenting to an emergency department with an acute asthma exacerbation; the intervention was theophylline with or without ethylene diamine, administered intravenously; the control was placebo, no drug or active comparator; the outcomes were forced expiratory volume in one second (FEV1), forced vital capacity (FVC), peak expiratory flow rate (PEFR), symptom scores, admission rates, duration of stay, rescue medication use, oxygen saturation, pulse rate, respiratory rate, or adverse events; the time was between 15 minutes and 48 hours after administration of theophylline; the setting was acute, inpatient treatment in a hospital.

\section{Study selection and study quality}

Two authors independently assessed all citations at the title/ abstract level in EPPI-Reviewer 4. Disagreements between the authors were resolved by consensus. Two authors then examined the full texts of all included abstracts in EPPIReviewer 4. In addition to the previously mentioned PICOTS criteria, studies were only included if they were randomized, controlled trials.

The Cochrane tool for assessing the risk of bias in $\mathrm{RCTs}^{10}$ was used to assess study quality. Two investigators assessed the risk of bias according to random sequence generation, allocation concealment, blinding of participants and personnel, blinding of outcome assessment, attrition, selective reporting, and other bias. We did not exclude studies if they were not blinded, but planned a sensitivity analysis to test the importance of blinding in assessing the outcomes.

\section{Data extraction}

One investigator extracted data from all included studies. A second investigator confirmed the data extraction. Data that were not given in the text or in tables were extracted using WebPlotDigitizer. ${ }^{11}$ We extracted the data as given in the text. For the meta-analysis, we converted standard errors to standard deviations. Where more than one control was present, we extracted all study arms. If more than one study arm was used in an analysis, we avoided a unit of analysis error by dividing the number in the study arm by the number of study arms used in the analysis. 


\section{Statistical analysis}

Meta-analysis was done using Review Manager (RevMan 5.3). ${ }^{12}$ Mean differences and standardized mean differences with $95 \%$ confidence intervals (CIs) were calculated using an inverse variance model. ${ }^{12}$ Odds ratios with $95 \%$ CI were calculated using the Mantel-Haenszel statistical method. ${ }^{13}$ Because of differences in study design and participants, we used a random effects model for all analyses.

\section{Results}

All study results refer to "theophylline" whether or not it contained ethylene diamine. For a breakdown of which studies used which drug, please refer to the study characteristics given in the following section, as well as Table 1 .

\section{Study characteristics}

A total of 52 study arms from 42 individual trials were included in the meta-analysis (Figure 1, Table 1). Adults were studied in 29 study arms, ${ }^{14-36}$ with children the focus of 17 study arms. ${ }^{37-53}$ One study did not restrict the age of participants, ${ }^{54}$ and one study did not report the age of participants. ${ }^{55}$ Twenty-five study arms compared theophylline with an active control such as adrenaline, beta- 2 agonists, or leukotriene receptor antagonists, 21 compared theophylline with placebo, and two studies compared theophylline with no drug. Forty-eight study arms used theophylline with ethylene diamine; and four used theophylline without ethylene diamine. Only two studies were funded or partly funded by industry. All other studies were funded and carried out by university or hospital clinical teams. Blinding of some kind took place in 37 study arms, with blinding being unclear in 11 arms. All studies were carried out in both males and females.

\section{Quality of included studies}

The quality of included studies is given in Figure 2. In general, the risk of bias was unclear or low. Reporting of the method of randomization, allocation concealment, and study protocols was frequently missing. The lack of blinding in some studies led to an increase in the risk of bias to some degree.

\section{FEVI/FVC}

The FEV1 and FVC (after a full breath) are commonly measured outcomes for asthma studies. FEV1 can be measured in liters (L), or alternatively as a percent of the predicted value. In our analysis, the majority of the studies used liters to measure FEV1. We carried out a subgroup meta-analysis of FEV1 (L) by control type (Figure 3A). Intravenous (IV) theophylline was not significantly different from adrenaline ( $p=0.12)$, a leukotriene receptor antagonist $(p=0.81)$, or placebo $(p=0.07)$ in increasing FEV1, but was significantly worse than beta- 2 agonists (mean difference $[\mathrm{MD}]=-0.20 \mathrm{~L}$ [95\% CI: $-0.34,-0.07], p=0.002$ ). A pooled analysis of all active controls, however, also showed a small but significantly improved FEV1 in the control compared with theophylline $(\mathrm{MD}=-0.14 \mathrm{~L}$ [95\% CI: $-0.25,-0.02], p=0.001$; Figure 3B). Pooling of the six studies measuring FEV1 as a percent of predicted showed no difference between theophylline and control $(\mathrm{MD}=3.78$ [95\% CI: $-1.08,8.63], p=0.13$, data not shown). Seven studies (nine study arms) reported on FVC (Figure 3C). There was no difference in FVC between theophylline and control groups $(p=0.73)$.

\section{PEFR}

PEFR is another common measurement of lung function in asthmatics. As for FEV1, PEFR can be measured in L or as a percent of the predicted value. A subgroup meta-analysis of PEFR (L) was performed to determine if theophylline was effective at increasing PEFR in the short-term (30 minutes2 hours) or the longer-term (5 hours-24 hours) (Figure 4A). There were no significant differences between theophylline and control at either time point. A sensitivity analysis removing the placebo-controlled trials from this analysis did not alter the results (data not shown). When measured as a percent of predicted PEFR value (Figure 4B), neither the short-term studies ( 30 minutes -2 hours; $p=0.56$ ) nor the longer-term studies ( 5 hours -48 hours; $p=0.44$ ) showed any significant differences between theophylline and control groups.

\section{Heart rate}

During an asthma exacerbation, the heart rate increases to compensate for a reduction in oxygenation in the blood. Therefore, a lower heart rate, both immediately after the administration of medication as well as over the longer term, indicates that the medication is relieving the bronchoconstriction. In order to compare the effect of IV theophylline on heart rate, we undertook a subgroup meta-analysis by time after infusion (Figure 5A). In the short-term (30 minutes3 hours post-infusion), theophylline lowered the heart rate by 4.17 beats per minute (bpm) compared with control therapy, which was significant ( $p=0.02$ ). At longer-term time points (24-36 hours post-infusion), the difference in heart rate between IV theophylline and control treatments was similar 
Table I Characteristics of included studies

\begin{tabular}{|c|c|c|c|c|c|c|c|c|c|c|}
\hline Study ID & $\begin{array}{l}\text { Intervention } \\
\text { (N) }\end{array}$ & $\begin{array}{l}\text { Control } \\
\text { (N) }\end{array}$ & $\begin{array}{l}\text { Study } \\
\text { type }\end{array}$ & $\begin{array}{l}\text { Study } \\
\text { length }\end{array}$ & Population & $\begin{array}{l}\text { Age } \\
\text { range, } \\
\text { years }\end{array}$ & $\begin{array}{l}\text { Average } \\
\text { age, } \\
\text { years }\end{array}$ & $\begin{array}{l}\text { Inclusion } \\
\text { (severity) }\end{array}$ & Intervention & Bolus dose \\
\hline $\begin{array}{l}\text { Anantharaman } \\
(1993 / I)^{14}\end{array}$ & 27 & 27 & $P$ & 30 minutes & Adults & $15-40$ & 28 & All & A & $250 \mathrm{mg}$ \\
\hline $\begin{array}{l}\text { Anantharaman } \\
(1993 / 2)^{14}\end{array}$ & 27 & 17 & $\mathrm{P}$ & 30 minutes & Adults & $15-40$ & 27 & All & $A$ & $250 \mathrm{mg}$ \\
\hline $\begin{array}{l}\text { Appel and Shim } \\
(1981)^{15}\end{array}$ & 12 & 12 & $P$ & 60 minutes & Adults & No data & 33 & All & A & $6 \mathrm{mg} / \mathrm{kg}$ \\
\hline $\begin{array}{l}\text { Coleridge } \\
\text { et al }(1993 / 1) \\
\text { (discharged) }^{16}\end{array}$ & 16 & 15 & $\mathrm{P}$ & 50 hours & Adults & No data & 34 & $\begin{array}{l}\text { Not recovered } \\
\text { at } 30 \text { minutes } \\
\text { after salbutamol }\end{array}$ & A & Not stated \\
\hline $\begin{array}{l}\text { Coleridge } \\
\text { et al }(1993 / 2) \\
\text { (inpatients) }^{16}\end{array}$ & 14 & 14 & $\mathrm{P}$ & 50 hours & Adults & No data & 34 & $\begin{array}{l}\text { Not recovered } \\
\text { at } 30 \text { minutes } \\
\text { after salbutamol }\end{array}$ & $\mathrm{A}$ & Not stated \\
\hline $\begin{array}{l}\text { Emerman et al } \\
(1986)^{17}\end{array}$ & 20 & 20 & $\mathrm{P}$ & 90 minutes & Adults & $18-45$ & 31 & All & A & $5.6 \mathrm{mg} / \mathrm{kg}$ \\
\hline $\begin{array}{l}\text { Evans et al } \\
(1980)^{18}\end{array}$ & 6 & 7 & $\mathrm{P}$ & 24 hours & Adults & No data & 28 & All & A & $\begin{array}{l}0.285 \mathrm{mg} / \mathrm{kg} / \\
\min \end{array}$ \\
\hline $\begin{array}{l}\text { Fanta et al } \\
(1986 / 1)^{19}\end{array}$ & 17 & 38 & $\mathrm{P}$ & 60 minutes & Adults & No data & 30 & All & A & $5.6 \mathrm{mg} / \mathrm{kg}$ \\
\hline $\begin{array}{l}\text { Fanta et al } \\
(1986 / 2)^{19}\end{array}$ & 17 & 41 & $P$ & 60 minutes & Adults & No data & 30 & All & A & $5.6 \mathrm{mg} / \mathrm{kg}$ \\
\hline $\begin{array}{l}\text { Femi-Pearse } \\
\text { et al }(1977 / I)^{20}\end{array}$ & 8 & 10 & $\mathrm{P}$ & 40 minutes & Adults & No data & No data & Not stated & A & $\begin{array}{l}250 \mathrm{mg} \text { over } \\
15 \text { minutes }\end{array}$ \\
\hline $\begin{array}{l}\text { Femi-Pearse } \\
\text { et al }(1977 / 2)^{20}\end{array}$ & 15 & 17 & $\mathrm{P}$ & 40 minutes & Adults & No data & No data & Not stated & A & $\begin{array}{l}250 \mathrm{mg} \text { over } \\
15 \text { minutes }\end{array}$ \\
\hline $\begin{array}{l}\text { Greif et al } \\
(1985)^{21}\end{array}$ & 10 & II & $\mathrm{P}$ & 120 minutes & Adults & $15-68$ & 38 & All & A & $6 \mathrm{mg} / \mathrm{kg}$ \\
\hline $\begin{array}{l}\text { Huang et al } \\
(1993)^{22}\end{array}$ & 10 & II & $\mathrm{P}$ & 48 hours & Adults & $22-48$ & 33 & Failed albuterol & A & $\begin{array}{l}\text { To achieve } \\
\text { I5 } \mu \mathrm{g} / \mathrm{mL}\end{array}$ \\
\hline $\begin{array}{l}\text { Johnson et al } \\
(1978)^{23}\end{array}$ & 19 & 20 & $\mathrm{P}$ & 36 hours & Adults & $16-65$ & 39 & $\begin{array}{l}\text { Requiring } \\
\text { treatment } \\
\text { after } 5 \mathrm{mg} / \mathrm{kg} \\
\text { theophylline } \\
\text { and nebulized } \\
\text { salbutamol }\end{array}$ & A & $5 \mathrm{mg} / \mathrm{kg}$ \\
\hline $\begin{array}{l}\text { Lindholm } \\
\text { and Helander } \\
(1966 / 1)^{24}\end{array}$ & 29 & 21 & $\mathrm{P}$ & 30 minutes & Adults & $22-73$ & 48 & $\begin{array}{l}\text { Moderate } \\
\text { severity }\end{array}$ & A & None \\
\hline $\begin{array}{l}\text { Lindholm } \\
\text { and Helander } \\
(1966 / 2)^{24}\end{array}$ & 29 & 23 & $\mathrm{P}$ & 30 minutes & Adults & $15-73$ & 49 & $\begin{array}{l}\text { Moderate } \\
\text { severity }\end{array}$ & A & None \\
\hline $\begin{array}{l}\text { Lindholm } \\
\text { and Helander } \\
(1966 / 3)^{24}\end{array}$ & 29 & 19 & $\mathrm{P}$ & 30 minutes & Adults & I5-73 & 49 & $\begin{array}{l}\text { Moderate } \\
\text { severity }\end{array}$ & A & None \\
\hline $\begin{array}{l}\text { Montserrat et al } \\
(1995)^{25}\end{array}$ & 6 & 6 & $\mathrm{P}$ & 51 hours & Adults & $21-62$ & $4 I$ & $\begin{array}{l}\text { Failed } \\
\text { bronchodilator } \\
\text { therapy }\end{array}$ & A & $6 \mathrm{mg} / \mathrm{kg}$ \\
\hline $\begin{array}{l}\text { Murphy et al } \\
(1993)^{26}\end{array}$ & 22 & 22 & $P$ & 5 hours & Adults & $18-45$ & 28 & $\begin{array}{l}\text { Failed } \\
\text { metaproterenol } \\
\text { sulfate }\end{array}$ & A & $8 \mathrm{mg} / \mathrm{kg}$ \\
\hline $\begin{array}{l}\text { Nakano et al } \\
(2006)^{27}\end{array}$ & 10 & 8 & $\mathrm{P}$ & Unclear & Adults & $22-70$ & 47 & $\begin{array}{l}\text { Only mild } \\
\text { to moderate } \\
\text { asthmatics } \\
\text { included }\end{array}$ & A & $\begin{array}{l}\text { To achieve } \\
\text { I } 8 \mu \mathrm{g} / \mathrm{mL}\end{array}$ \\
\hline
\end{tabular}




\begin{tabular}{|c|c|c|c|c|c|c|c|c|}
\hline Ongoing dose & Control & Bolus dose & $\begin{array}{l}\text { Ongoing } \\
\text { dose }\end{array}$ & $\begin{array}{l}\text { Background } \\
\text { medication }\end{array}$ & Gender & Country & Blinding & Funding \\
\hline None & $\begin{array}{l}\text { Adrenaline } \\
\text { (sc) }\end{array}$ & $\mathrm{Img}$ & None & Oxygen & Mixed & Singapore & Unclear & Hospital \\
\hline None & $\begin{array}{l}\text { Salbutamol } \\
\text { (nebulized) }\end{array}$ & $10 \mathrm{mg}$ & None & Oxygen & Mixed & Singapore & Unclear & Hospital \\
\hline None & $\begin{array}{l}\text { Epinephrine } \\
\text { (sc) }\end{array}$ & $0.3-0.5 \mathrm{~mL}$ & None & None & Mixed & USA & $\begin{array}{l}\text { Double } \\
\text { blind }\end{array}$ & $\begin{array}{l}\text { University/ } \\
\text { hospital }\end{array}$ \\
\hline $0.5-0.75 \mathrm{mg} / \mathrm{kg} / \mathrm{h}$ & Placebo & $\mathrm{N} / \mathrm{A}$ & N/A & $\begin{array}{l}\text { Hydrocortisone (IV), } \\
\text { salbutamol (neb), } \\
\text { ipratropiumbromide(neb) }\end{array}$ & Mixed & Australia & $\begin{array}{l}\text { Double } \\
\text { blind }\end{array}$ & Hospital \\
\hline $0.5-0.75 \mathrm{mg} / \mathrm{kg} / \mathrm{h}$ & Placebo & N/A & $\mathrm{N} / \mathrm{A}$ & $\begin{array}{l}\text { Hydrocortisone (IV), } \\
\text { salbutamol (neb), } \\
\text { ipratropium bromide } \\
\text { (neb) }\end{array}$ & Mixed & Australia & $\begin{array}{l}\text { Double } \\
\text { blind }\end{array}$ & Hospital \\
\hline None & $\begin{array}{l}\text { Epinephrine } \\
\text { (sc) }\end{array}$ & $0.3 \mathrm{~mL}$ & None & None & Mixed & USA & $\begin{array}{l}\text { Double } \\
\text { blind }\end{array}$ & $\begin{array}{l}\text { University/ } \\
\text { hospital }\end{array}$ \\
\hline $0.014 \mathrm{mg} / \mathrm{kg} / \mathrm{min}$ & Salbutamol IV & $0.285 \mu \mathrm{g} / \mathrm{kg} / \mathrm{min}$ & $0.057 \mu \mathrm{g} / \mathrm{kg} / \mathrm{min}$ & $\begin{array}{l}\text { Hydrocortisone (IV), } \\
\text { potassium chloride (IV) }\end{array}$ & Mixed & UK & $\begin{array}{l}\text { Single } \\
\text { blind }\end{array}$ & $\begin{array}{l}\text { University/ } \\
\text { hospital }\end{array}$ \\
\hline $0.9 \mathrm{mg} / \mathrm{kg} / \mathrm{h}$ & $\begin{array}{l}\text { Epinephrine } \\
\text { (sc) }\end{array}$ & $\begin{array}{l}0.3 \mathrm{mg} \text { at } \\
20 \mathrm{~min} \times 3\end{array}$ & None & Supplemental oxygen & Mixed & USA & Unclear & $\begin{array}{l}\text { University/ } \\
\text { hospital }\end{array}$ \\
\hline $0.9 \mathrm{mg} / \mathrm{kg} / \mathrm{h}$ & $\begin{array}{l}\text { Isoproterenol } \\
\text { (nebulized) }\end{array}$ & $\begin{array}{l}2.5 \mathrm{mg} \text { at } \\
20 \mathrm{~min} \times 3\end{array}$ & None & Supplemental oxygen & Mixed & USA & Unclear & $\begin{array}{l}\text { University/ } \\
\text { hospital }\end{array}$ \\
\hline None & Salbutamol IV & $200 \mu \mathrm{g}$ bolus & None & Not stated & $\begin{array}{l}\text { Not } \\
\text { stated }\end{array}$ & Nigeria & $\begin{array}{l}\text { Single } \\
\text { blind }\end{array}$ & University \\
\hline None & Salbutamol IV & $\begin{array}{l}200 \mu \mathrm{g} \text { over } \\
15 \text { minutes }\end{array}$ & None & Not stated & $\begin{array}{l}\text { Not } \\
\text { stated }\end{array}$ & Nigeria & $\begin{array}{l}\text { Double } \\
\text { blind }\end{array}$ & University \\
\hline None & Salbutamol IV & $4 \mu g / \mathrm{kg}$ & None & Not stated & Mixed & Israel & $\begin{array}{l}\text { Single } \\
\text { blind }\end{array}$ & $\begin{array}{l}\text { University/ } \\
\text { hospital }\end{array}$ \\
\hline $0.6 \mathrm{mg} / \mathrm{kg} / \mathrm{h}$ & Placebo & $\mathrm{N} / \mathrm{A}$ & $\mathrm{N} / \mathrm{A}$ & $\begin{array}{l}\text { Albuterol (neb), } \\
\text { methylprednisone (IV) }\end{array}$ & Mixed & USA & $\begin{array}{l}\text { Double } \\
\text { blind }\end{array}$ & University \\
\hline $\mathrm{l} \mathrm{mg} / \mathrm{min}$ & Salbutamol IV & None & $10 \mu \mathrm{g} / \mathrm{min}$ & $\begin{array}{l}\text { Bolus aminophyllyine, } \\
\text { salbutamol (neb), } \\
\text { hydrocortisone IV, } \\
\text { prednisone (oral) }\end{array}$ & Mixed & UK & Unclear & Hospital \\
\hline $250 \mathrm{mg}$ & $\begin{array}{l}\text { Adrenaline } \\
\text { (sc) }\end{array}$ & None & $0.5 \mathrm{mg}$ & Not stated & Mixed & Sweden & $\begin{array}{l}\text { Double } \\
\text { blind }\end{array}$ & University \\
\hline $250 \mathrm{mg}$ & Isoprenaline & None & $\begin{array}{l}0.06 \mathrm{mg} \text { inhaled } \\
\text { three times }\end{array}$ & Not stated & Mixed & Sweden & $\begin{array}{l}\text { Double } \\
\text { blind }\end{array}$ & University \\
\hline $250 \mathrm{mg}$ & Placebo & $N / A$ & $N / A$ & Not stated & Mixed & Sweden & $\begin{array}{l}\text { Double } \\
\text { blind }\end{array}$ & University \\
\hline $0.9 \mathrm{mg} / \mathrm{kg} / \mathrm{h}$ & Placebo & N/A & N/A & $\begin{array}{l}\text { Salbutamol, } \\
\text { corticosteroids, oxygen }\end{array}$ & Mixed & Spain & $\begin{array}{l}\text { Double } \\
\text { blind }\end{array}$ & University \\
\hline $0.8 \mathrm{mg} / \mathrm{kg} / \mathrm{h}$ & Placebo & N/A & $\mathrm{N} / \mathrm{A}$ & Methylprednisolone (IV) & & USA & $\begin{array}{l}\text { Double } \\
\text { blind }\end{array}$ & $\begin{array}{l}\text { University/ } \\
\text { hospital }\end{array}$ \\
\hline None & $\begin{array}{l}\text { Salbutamol } \\
\text { (nebulized) }\end{array}$ & $2 \mathrm{mg}$ & None & None & Mixed & Japan & Unclear & $\begin{array}{l}\text { University/ } \\
\text { hospital }\end{array}$ \\
\hline
\end{tabular}


Table I (Continued)

\begin{tabular}{|c|c|c|c|c|c|c|c|c|c|c|}
\hline Study ID & $\begin{array}{l}\text { Intervention } \\
\text { (N) }\end{array}$ & $\begin{array}{l}\text { Control } \\
\text { (N) }\end{array}$ & $\begin{array}{l}\text { Study } \\
\text { type }\end{array}$ & $\begin{array}{l}\text { Study } \\
\text { length }\end{array}$ & Population & $\begin{array}{l}\text { Age } \\
\text { range, } \\
\text { years }\end{array}$ & $\begin{array}{l}\text { Average } \\
\text { age, } \\
\text { years }\end{array}$ & $\begin{array}{l}\text { Inclusion } \\
\text { (severity) }\end{array}$ & Intervention & Bolus dose \\
\hline $\begin{array}{l}\text { NCT00442338 } \\
(2007)^{28}\end{array}$ & 31 & 30 & $P$ & 60 minutes & Adults & No data & 56 & All & $A$ & None \\
\hline $\begin{array}{l}\text { Rodrigo and } \\
\text { Rodrigo (1994) }\end{array}$ & 45 & 49 & $P$ & 120 minutes & Adults & No data & 36 & All & $\mathrm{A}$ & $5.6 \mathrm{mg} / \mathrm{kg}$ \\
\hline $\begin{array}{l}\text { Rossing et al } \\
(1980 / 1)^{30}\end{array}$ & 17 & 16 & $P$ & 60 minutes & Adults & $18-45$ & 30 & All & $\mathrm{A}$ & $5.6 \mathrm{mg} / \mathrm{kg}$ \\
\hline $\begin{array}{l}\text { Rossing et al } \\
(1980 / 2)^{30}\end{array}$ & 17 & 15 & $P$ & 60 minutes & Adults & $18-45$ & 31 & All & $\mathrm{A}$ & $5.6 \mathrm{mg} / \mathrm{kg}$ \\
\hline $\begin{array}{l}\text { Self et al } \\
(1990)^{31}\end{array}$ & 21 & 18 & $P$ & 32 hours & Adults & $18-49$ & 32 & $\begin{array}{l}\text { Failed } \\
\text { albuterol and } \\
\text { corticosteroids }\end{array}$ & $\mathrm{A}$ & $\begin{array}{l}\text { To achieve } \\
\text { I0-20 } \mu \mathrm{g} / \mathrm{mL}\end{array}$ \\
\hline $\begin{array}{l}\text { Sharma et al } \\
(1984 / I)^{32}\end{array}$ & 10 & 10 & $\mathrm{P}$ & 3 hours & Adults & No data & 33 & $\begin{array}{l}\text { No broncho- } \\
\text { dilators in } \\
\text { previous } \\
24 \text { hours }\end{array}$ & $\mathrm{A}$ & $250 \mathrm{mg}$ \\
\hline $\begin{array}{l}\text { Sharma et al } \\
(1984 / 2)^{32}\end{array}$ & 10 & 10 & $P$ & 3 hours & Adults & No data & 32 & $\begin{array}{l}\text { No broncho- } \\
\text { dilators in } \\
\text { previous } \\
24 \text { hours }\end{array}$ & $\mathrm{A}$ & $250 \mathrm{mg}$ \\
\hline $\begin{array}{l}\text { Siegel et al } \\
(1985)^{33}\end{array}$ & 20 & 20 & $P$ & 3 hours & Adults & $18-45$ & 30 & None & $A$ & $5.6 \mathrm{mg} / \mathrm{kg}$ \\
\hline $\begin{array}{l}\text { Tribe et al } \\
(1976)^{34}\end{array}$ & 12 & II & $P$ & 3 hours & Adults & $17-78$ & 44 & All & $\mathrm{A}$ & $250 \mathrm{mg}$ \\
\hline $\begin{array}{l}\text { Wrenn et al } \\
(1991)^{35}\end{array}$ & 32 & 35 & $P$ & 120 minutes & Adults & $\begin{array}{l}16 \text { or } \\
\text { older }\end{array}$ & 34 & All & $\mathrm{A}$ & $5.6 \mathrm{mg} / \mathrm{kg}$ \\
\hline $\begin{array}{l}\text { Zainudin et al } \\
(1994)^{36}\end{array}$ & II & 14 & $\mathrm{P}$ & 48 hours & Adults & $18-60$ & No data & Severe asthma & $\mathrm{A}$ & No bolus \\
\hline $\begin{array}{l}\text { Bien et al } \\
(1995)^{37}\end{array}$ & 19 & 20 & $P$ & 24 hours & Children & $2-10$ & 6 & $\begin{array}{l}\text { Excluded: ICU } \\
\text { admission, use } \\
\text { of systemic } \\
\text { corticosteroids }\end{array}$ & $\mathrm{T}$ & $1.6 \mathrm{mg} / \mathrm{mL}$ \\
\hline $\begin{array}{l}\text { Carter et al } \\
(1993)^{38}\end{array}$ & 12 & 9 & $P$ & 36 hours & Children & $5-18$ & 12 & Failed albuterol & A & $\begin{array}{l}\text { To achieve } \\
\text { I5 } 5 \mathrm{~g} / \mathrm{mL}\end{array}$ \\
\hline $\begin{array}{l}\text { D'Avila et al } \\
(2008)^{39}\end{array}$ & 30 & 30 & $P$ & 60 minutes & Children & $2-5$ & 3 & $\begin{array}{l}\text { Failed } \\
\text { albuterol and } \\
\text { corticosteroids }\end{array}$ & $A$ & $\begin{array}{l}5 \mathrm{mg} / \mathrm{kg} \text { in } \\
\text { two boluses } \\
6 \text { hours } \\
\text { apart }\end{array}$ \\
\hline $\begin{array}{l}\text { DiGiulio et al } \\
(1993)^{40}\end{array}$ & 16 & 13 & $P$ & 35 hours & Children & $2-16$ & 7 & Failed albuterol & $\mathrm{T}$ & $4.8 \mathrm{mg} / \mathrm{kg}$ \\
\hline $\begin{array}{l}\text { Hambleton and } \\
\text { Stone }(1979)^{41}\end{array}$ & 9 & 9 & $P$ & 24 hours & Children & $1.5-7$ & No data & $\begin{array}{l}\text { Requiring } \\
\text { intense hospital } \\
\text { treatment }\end{array}$ & $\mathrm{A}$ & $4 \mathrm{mg} / \mathrm{kg}$ \\
\hline $\begin{array}{l}\text { Ibrahim et al } \\
(1993 / 1)^{42}\end{array}$ & 40 & 40 & $P$ & 120 minutes & Children & No data & 10 & $\begin{array}{l}\text { No broncho- } \\
\text { dilators in } \\
\text { previous } \\
12 \text { hours }\end{array}$ & $A$ & $5 \mathrm{mg} / \mathrm{kg}$ \\
\hline $\begin{array}{l}\text { Ibrahim et al } \\
(1993 / 2)^{42}\end{array}$ & 40 & 40 & $P$ & 120 minutes & Children & No data & 10 & $\begin{array}{l}\text { No broncho- } \\
\text { dilators in } \\
\text { previous } \\
12 \text { hours }\end{array}$ & A & $5 \mathrm{mg} / \mathrm{kg}$ \\
\hline $\begin{array}{l}\text { Needleman et al } \\
(1995)^{43}\end{array}$ & 25 & 20 & $\mathrm{P}$ & 120 minutes & Children & $2-18$ & 8 & Failed albuterol & $\mathrm{T}$ & $6-8 \mathrm{mg} / \mathrm{kg}$ \\
\hline $\begin{array}{l}\text { Nuhoglu et al } \\
(1998)^{44}\end{array}$ & 17 & 19 & $P$ & 24 hours & Children & $2-16$ & 6 & All & $A$ & $6 \mathrm{mg} / \mathrm{kg}$ \\
\hline
\end{tabular}




\begin{tabular}{|c|c|c|c|c|c|c|c|c|}
\hline Ongoing dose & Control & Bolus dose & $\begin{array}{l}\text { Ongoing } \\
\text { dose }\end{array}$ & $\begin{array}{l}\text { Background } \\
\text { medication }\end{array}$ & Gender & Country & Blinding & Funding \\
\hline $250 \mathrm{mg}$ & Montelukast & None & $14 \mathrm{mg}$ & $\begin{array}{l}\text { Inhaled beta-agonist or } \\
\text { oxygen }\end{array}$ & Mixed & Multicenter & Unclear & Industry \\
\hline $0.9 \mathrm{mg} / \mathrm{kg} / \mathrm{h}$ & Placebo & N/A & N/A & $\begin{array}{l}\text { Salbutamol (neb), } \\
\text { hydrocortisone (IV) }\end{array}$ & Mixed & Uruguay & $\begin{array}{l}\text { Double } \\
\text { blind }\end{array}$ & $\begin{array}{l}\text { University/ } \\
\text { hospital }\end{array}$ \\
\hline $0.9 \mathrm{mg} / \mathrm{kg} / \mathrm{h}$ & $\begin{array}{l}\text { Epinephrine } \\
\text { (sc) }\end{array}$ & $0.3 \mathrm{~mL} \times 3$ & None & Oxygen & Mixed & USA & Unclear & $\begin{array}{l}\text { University/ } \\
\text { hospital }\end{array}$ \\
\hline $0.9 \mathrm{mg} / \mathrm{kg} / \mathrm{h}$ & $\begin{array}{l}\text { Isoproterenol } \\
\text { (neb) }\end{array}$ & $2.5 \mathrm{mg} \times 3$ & None & Oxygen & Mixed & USA & Unclear & $\begin{array}{l}\text { University/ } \\
\text { hospital }\end{array}$ \\
\hline $\begin{array}{l}\text { To achieve } \\
\text { I0-20 } \mu \mathrm{g} / \mathrm{mL}\end{array}$ & Placebo & N/A & $N / A$ & $\begin{array}{l}\text { Prednisone (oral), } \\
\text { albuterol (neb), oxygen }\end{array}$ & Mixed & USA & $\begin{array}{l}\text { Double } \\
\text { blind }\end{array}$ & $\begin{array}{l}\text { Industry/ } \\
\text { university }\end{array}$ \\
\hline None & Salbutamol & $250 \mu \mathrm{g}$ & None & None & Mixed & India & Unclear & Hospital \\
\hline None & Terbutaline & $250 \mu \mathrm{g}$ & None & None & Mixed & India & Unclear & Hospital \\
\hline $0.7 \mathrm{mg} / \mathrm{kg} / \mathrm{h}$ & Placebo & N/A & N/A & Meteproterenol & Mixed & USA & $\begin{array}{l}\text { Double } \\
\text { blind }\end{array}$ & $\begin{array}{l}\text { University/ } \\
\text { hospital }\end{array}$ \\
\hline None & Salbutamol IV & $100 \mu \mathrm{g}$ & None & Hydrocortisone (IV) & Mixed & Australia & $\begin{array}{l}\text { Double } \\
\text { blind }\end{array}$ & Hospital \\
\hline $0.9 \mathrm{mg} / \mathrm{kg} / \mathrm{h}$ & Placebo & $\mathrm{N} / \mathrm{A}$ & $\mathrm{N} / \mathrm{A}$ & $\begin{array}{l}\text { Methylprednisolone (IV), } \\
\text { metaproterenol (neb) }\end{array}$ & Mixed & USA & $\begin{array}{l}\text { Double } \\
\text { blind }\end{array}$ & University \\
\hline $0.6-0.9 \mathrm{mg} / \mathrm{kg} / \mathrm{h}$ & No infusion & N/A & N/A & $\begin{array}{l}\text { Salbutamol (neb), } \\
\text { hydrocortisone (IV), oral } \\
\text { prednisolone, oxygen }\end{array}$ & Mixed & Malaysia & Not blind & University \\
\hline $\begin{array}{l}\text { To achieve } \\
\text { I0-20 } \mu \mathrm{g} / \mathrm{mL}\end{array}$ & Placebo & $\mathrm{N} / \mathrm{A}$ & $\mathrm{N} / \mathrm{A}$ & $\begin{array}{l}\text { Albuterol (neb), } \\
\text { methylprednisone (IV), } \\
\text { oxygen }\end{array}$ & Mixed & USA & $\begin{array}{l}\text { Double } \\
\text { blind }\end{array}$ & Hospital \\
\hline $1 \mathrm{mg} / \mathrm{kg}$ & Placebo & $\mathrm{N} / \mathrm{A}$ & $\mathrm{N} / \mathrm{A}$ & $\begin{array}{l}\text { Albuterol (neb), } \\
\text { methylprednisone (IV), } \\
\text { oxygen }\end{array}$ & Mixed & USA & $\begin{array}{l}\text { Double } \\
\text { blind }\end{array}$ & $\begin{array}{l}\text { University/ } \\
\text { hospital }\end{array}$ \\
\hline None & Placebo & N/A & N/A & $\begin{array}{l}\text { Prednisolone or } \\
\text { prednisone I mg/kg, } \\
\text { albuterol } 150 \mu g / k g\end{array}$ & Mixed & Brazil & $\begin{array}{l}\text { Double } \\
\text { blind }\end{array}$ & $\begin{array}{l}\text { University/ } \\
\text { hospital }\end{array}$ \\
\hline $\begin{array}{l}\text { To achieve } \\
12-20 \mathrm{mg} / \mathrm{L}\end{array}$ & Placebo & N/A & $\mathrm{N} / \mathrm{A}$ & $\begin{array}{l}\text { Methylprednisolone (IV), } \\
\text { albuterol (neb) }\end{array}$ & Mixed & USA & $\begin{array}{l}\text { Double } \\
\text { blind }\end{array}$ & $\begin{array}{l}\text { University/ } \\
\text { hospital }\end{array}$ \\
\hline $0.6 \mathrm{mg} / \mathrm{kg} / \mathrm{h}$ & Salbutamol IV & $4 \mu g / \mathrm{kg}$ & $0.6 \mu \mathrm{g} / \mathrm{kg} / \mathrm{h}$ & Hydrocortisone (IV) & Mixed & UK & $\begin{array}{l}\text { Double } \\
\text { blind }\end{array}$ & Hospital \\
\hline None & Adrenaline & $0.01 \mathrm{mg} / \mathrm{kg}$ & None & None & Mixed & Sudan & Unclear & $\begin{array}{l}\text { University/ } \\
\text { hospital }\end{array}$ \\
\hline None & Salbutamol & $0.15 \mathrm{mg} / \mathrm{kg}$ & None & None & Mixed & Sudan & Unclear & $\begin{array}{l}\text { University/ } \\
\text { hospital }\end{array}$ \\
\hline $0.8-1.0 \mathrm{mg} / \mathrm{kg} / \mathrm{h}$ & Placebo & $\mathrm{N} / \mathrm{A}$ & N/A & $\begin{array}{l}\text { Methylprednisolone (IV), } \\
\text { albuterol (neb) }\end{array}$ & Mixed & USA & $\begin{array}{l}\text { Double } \\
\text { blind }\end{array}$ & $\begin{array}{l}\text { University/ } \\
\text { hospital }\end{array}$ \\
\hline $1 \mathrm{mg} / \mathrm{kg} / \mathrm{h}$ & Placebo & N/A & N/A & $\begin{array}{l}\text { Methylprednisolone (IV), } \\
\text { salbutamol }\end{array}$ & Mixed & Turkey & $\begin{array}{l}\text { Double } \\
\text { blind }\end{array}$ & $\begin{array}{l}\text { University/ } \\
\text { hospital }\end{array}$ \\
\hline
\end{tabular}


Table I (Continued)

\begin{tabular}{|c|c|c|c|c|c|c|c|c|c|c|}
\hline Study ID & $\begin{array}{l}\text { Intervention } \\
\text { (N) }\end{array}$ & $\begin{array}{l}\text { Control } \\
\text { (N) }\end{array}$ & $\begin{array}{l}\text { Study } \\
\text { type }\end{array}$ & $\begin{array}{l}\text { Study } \\
\text { length }\end{array}$ & Population & $\begin{array}{l}\text { Age } \\
\text { range, } \\
\text { years }\end{array}$ & $\begin{array}{l}\text { Average } \\
\text { age, } \\
\text { years }\end{array}$ & $\begin{array}{l}\text { Inclusion } \\
\text { (severity) }\end{array}$ & Intervention & Bolus dose \\
\hline $\begin{array}{l}\text { Pierson et al } \\
(197 I)^{45}\end{array}$ & 11 & 12 & $P$ & 24 hours & Children & $5-17$ & 12 & $\begin{array}{l}\text { Failed } \\
\text { epinephrine }\end{array}$ & $A$ & $7 \mathrm{mg} / \mathrm{kg}$ \\
\hline $\begin{array}{l}\text { Ream et al } \\
(2001)^{46}\end{array}$ & 23 & 24 & $P$ & 48 hours & Children & No data & 9 & $\begin{array}{l}\text { Severe asthma, } \\
\text { failed repeated } \\
\text { albuterol } \\
\text { nebulizations }\end{array}$ & A & $\begin{array}{l}\text { To achieve } \\
\text { 12-17 } \mu g \cdot \mathrm{mL}\end{array}$ \\
\hline $\begin{array}{l}\text { Roberts et al } \\
(2003)^{47}\end{array}$ & 26 & 18 & $P$ & 120 minutes & Children & $\begin{array}{l}\text { I.19-I5.55 } \\
(\mathrm{IQR})\end{array}$ & 4 & $\begin{array}{l}\text { Failed } \\
\text { salbutamol and } \\
\text { ipratropium }\end{array}$ & A & $5 \mathrm{mg} / \mathrm{kg}$ \\
\hline $\begin{array}{l}\text { Singhi et al } \\
(20 / 4 / I)^{48}\end{array}$ & 33 & 34 & $P$ & 60 minutes & Children & $1-12$ & 5 & $\begin{array}{l}\text { Failed } \\
\text { salbutamol, } \\
\text { budesonide, } \\
\text { ipratropium } \\
\text { bromide, and } \\
\text { hydrocortisone }\end{array}$ & $A$ & $5 \mathrm{mg} / \mathrm{kg}$ \\
\hline $\begin{array}{l}\text { Singhi et al } \\
(20 \mid 4 / 2)^{48}\end{array}$ & 33 & 33 & $P$ & 60 minutes & Children & $1-12$ & 4 & $\begin{array}{l}\text { Failed } \\
\text { salbutamol, } \\
\text { budesonide, } \\
\text { ipratropium } \\
\text { bromide, and } \\
\text { hydrocortisone }\end{array}$ & A & $5 \mathrm{mg} / \mathrm{kg}$ \\
\hline $\begin{array}{l}\text { Strauss et al } \\
(1994)^{49}\end{array}$ & 14 & 17 & $P$ & 48 hours & Children & $5-18$ & 11 & Failed albuterol & $A$ & $7 \mathrm{mg} / \mathrm{kg}$ \\
\hline $\begin{array}{l}\text { Tiwari et al } \\
(2016)^{50}\end{array}$ & 24 & 24 & $P$ & 24 hours & Children & $1-12$ & 4 & $\begin{array}{l}\text { Moderate to } \\
\text { severe asthma }\end{array}$ & $A$ & $5 \mathrm{mg} / \mathrm{kg}$ \\
\hline $\begin{array}{l}\text { Vieira et al } \\
(2000)^{5 !}\end{array}$ & 24 & 19 & $P$ & 24 hours & Children & $\mathrm{I}-7$ & 6 & $\begin{array}{l}\text { Wood-Downes } \\
\text { score 3-6 after } \\
\text { three fenoterol } \\
\text { nebulizations }\end{array}$ & A & $6 \mathrm{mg} / \mathrm{kg}$ \\
\hline $\begin{array}{l}\text { Wheeler et al } \\
(2005)^{52}\end{array}$ & 13 & 16 & $P$ & 24 hours & Children & $3-15$ & 9 & $\begin{array}{l}\text { Severe asthma, } \\
\text { CAS } \geq 7\end{array}$ & $\mathrm{~T}$ & $6.4 \mathrm{mg} / \mathrm{kg}$ \\
\hline $\begin{array}{l}\text { Yung and South } \\
(1998)^{53}\end{array}$ & 81 & 82 & $P$ & 24 hours & Children & $1-19$ & 6 & $\begin{array}{l}\text { Failed } \\
\text { salbutamol }\end{array}$ & A & $10 \mathrm{mg} / \mathrm{kg}$ \\
\hline $\begin{array}{l}\text { Whig et al } \\
(2001)^{54}\end{array}$ & 20 & 20 & $P$ & 13 hours & Both & $2-25$ & No data & $\begin{array}{l}\text { Failed one } \\
\text { dose of } \\
\text { salbutamol plus } \\
\text { hydrocortisone } \\
4 \mathrm{mg} / \mathrm{kg}\end{array}$ & $A$ & $6 \mathrm{mg} / \mathrm{kg}$ \\
\hline $\begin{array}{l}\text { Williams et al } \\
(1975)^{55}\end{array}$ & 9 & 11 & $P$ & 60 minutes & Unclear & No data & No data & Severe asthma & $A$ & None \\
\hline
\end{tabular}

Abbreviations: A, theophylline with ethylene diamine (aminophylline); T, theophylline; P, parallel; sc, subcutaneous; N/A, not applicable; IV, intravenous; neb, nebulization; $\mathrm{IQR}$, interquartile range; CAS, Clinical Asthma Score.

$(-3.59 \mathrm{bpm})$, but failed to reach statistical significance $(p=0.32)$. In order to determine if theophylline was superior to other active therapies, we undertook a subgroup metaanalysis by control type (Figure 5B). In the active control studies, theophylline lowered the heart rate by $5.17 \mathrm{bpm}$ more than active controls, and this difference was significant $(p=0.01)$. In the placebo-controlled trials, no significant difference was noted $(p=0.79)$. 


\begin{tabular}{|c|c|c|c|c|c|c|c|c|}
\hline Ongoing dose & Control & Bolus dose & $\begin{array}{l}\text { Ongoing } \\
\text { dose }\end{array}$ & $\begin{array}{l}\text { Background } \\
\text { medication }\end{array}$ & Gender & Country & Blinding & Funding \\
\hline $9 \mathrm{mg} / \mathrm{kg} / 24 \mathrm{~h}$ & Placebo & $\mathrm{N} / \mathrm{A}$ & $\mathrm{N} / \mathrm{A}$ & $\begin{array}{l}\text { Epinephrine, } \\
\text { hydrocortisone, } \\
\text { oxygen, phenylephrine, } \\
\text { isoproterenol }\end{array}$ & Mixed & USA & $\begin{array}{l}\text { Double } \\
\text { blind }\end{array}$ & Hospital \\
\hline $0.5-0.8 \mathrm{mg} / \mathrm{kg} / \mathrm{h}$ & No infusion & $\mathrm{N} / \mathrm{A}$ & $\mathrm{N} / \mathrm{A}$ & $\begin{array}{l}\text { Albuterol (neb), } \\
\text { methylprednisone (IV), } \\
\text { ipratropium }\end{array}$ & Mixed & USA & $\begin{array}{l}\text { Partly } \\
\text { blind }\end{array}$ & Hospital \\
\hline $0.9 \mathrm{mg} / \mathrm{kg} / \mathrm{h}$ & Salbutamol IV & I5 $\mu \mathrm{g} / \mathrm{kg}$ & None & $\begin{array}{l}\text { Salbutamol (neb), } \\
\text { ipratropium (neb) }\end{array}$ & Mixed & UK & $\begin{array}{l}\text { Double } \\
\text { blind }\end{array}$ & Hospital \\
\hline $0.9 \mathrm{mg} / \mathrm{kg} / \mathrm{h}$ & $\begin{array}{l}\text { Magnesium } \\
\text { sulfate }\end{array}$ & $25 \mathrm{mg} / \mathrm{kg}$ & None & $\begin{array}{l}\text { Salbutamol (neb), } \\
\text { ipratropium (neb), } \\
\text { budesonide, } \\
\text { hydrocortisone }\end{array}$ & Mixed & India & $\begin{array}{l}\text { Partly } \\
\text { blind }\end{array}$ & $\begin{array}{l}\text { University/ } \\
\text { hospital }\end{array}$ \\
\hline $0.9 \mathrm{mg} / \mathrm{kg} / \mathrm{h}$ & Terbutaline & $10 \mu g / k g$ & $0.1 \mu \mathrm{g} / \mathrm{kg} / \mathrm{min}$ & $\begin{array}{l}\text { Salbutamol (neb), } \\
\text { ipratropium (neb), } \\
\text { budesonide, } \\
\text { hydrocortisone }\end{array}$ & Mixed & India & $\begin{array}{l}\text { Partly } \\
\text { blind }\end{array}$ & $\begin{array}{l}\text { University/ } \\
\text { hospital }\end{array}$ \\
\hline $25 \mathrm{mg} / \mathrm{kg} / \mathrm{h}$ & Placebo & $\mathrm{N} / \mathrm{A}$ & $\mathrm{N} / \mathrm{A}$ & $\begin{array}{l}\text { Albuterol (neb), } \\
\text { methylprednisone (IV, } \\
\text { oxygen) }\end{array}$ & Mixed & USA & $\begin{array}{l}\text { Double } \\
\text { blind }\end{array}$ & Hospital \\
\hline $0.9 \mathrm{mg} / \mathrm{kg} / \mathrm{h}$ & Ketamine & $0.5 \mathrm{mg} / \mathrm{kg}$ & $0.6 \mathrm{mg} / \mathrm{kg} / \mathrm{h}$ & $\begin{array}{l}\text { Salbutamol (neb), } \\
\text { ipratropium (neb), } \\
\text { hydrocortisone }\end{array}$ & Mixed & India & $\begin{array}{l}\text { Partly } \\
\text { blind }\end{array}$ & Hospital \\
\hline $1.2 \mathrm{mg} / \mathrm{kg} / \mathrm{h}$ & Placebo & N/A & $N / A$ & $\begin{array}{l}\text { Hydrocortisone (IV), } \\
\text { fenoterol (neb) }\end{array}$ & Mixed & Brazil & $\begin{array}{l}\text { Double } \\
\text { blind }\end{array}$ & $\begin{array}{l}\text { University/ } \\
\text { hospital }\end{array}$ \\
\hline $0.6-1.0 \mathrm{mg} / \mathrm{kg} / \mathrm{h}$ & Terbutaline & $20 \mu \mathrm{g} / \mathrm{kg}$ & $0.4 \mu \mathrm{g} / \mathrm{kg} / \mathrm{h}$ & $\begin{array}{l}\text { Methylprednisolone (IV), } \\
\text { albuterol (neb) }\end{array}$ & Mixed & USA & $\begin{array}{l}\text { Double } \\
\text { blind }\end{array}$ & $\begin{array}{l}\text { University/ } \\
\text { hospital }\end{array}$ \\
\hline $0.7-1.1 \mathrm{mg} / \mathrm{kg} / \mathrm{h}$ & Placebo & N/A & $\mathrm{N} / \mathrm{A}$ & $\begin{array}{l}\text { Methylprednisolone (IV), } \\
\text { salbutamol (neb), } \\
\text { ipratropium bromide } \\
\text { (neb) }\end{array}$ & Mixed & Australia & $\begin{array}{l}\text { Double } \\
\text { blind }\end{array}$ & Hospital \\
\hline $0.5 \mathrm{mg} / \mathrm{kg} / \mathrm{h}$ & Placebo & N/A & $N / A$ & $\begin{array}{l}\text { Hydrocortisone (IV), } \\
\text { Salbutamol (neb) }\end{array}$ & Mixed & India & Unclear & $\begin{array}{l}\text { University/ } \\
\text { hospital }\end{array}$ \\
\hline $0.5 \mathrm{~g}$ over I h & Salbutamol IV & None & $\begin{array}{l}500 \mu \mathrm{g} \text { over } \\
\mathrm{I} \mathrm{h}\end{array}$ & $\begin{array}{l}\text { Hydrocortisone (IV), } \\
\text { oxygen }\end{array}$ & $\begin{array}{l}\text { Not } \\
\text { stated }\end{array}$ & UK & $\begin{array}{l}\text { Double } \\
\text { blind }\end{array}$ & Hospital \\
\hline
\end{tabular}

\section{Respiratory rate}

An increased respiratory rate is, like heart rate, a sign of an ongoing asthma exacerbation..$^{56}$ Thus, a reduction in the respiratory rate should indicate an improvement in the status of a patient with acute asthma. We undertook a meta-analysis of the seven study arms measuring this outcome (Figure 6). Theophylline was slightly less effective at lower respiratory rate, although this was not significant $(p=0.08)$. 


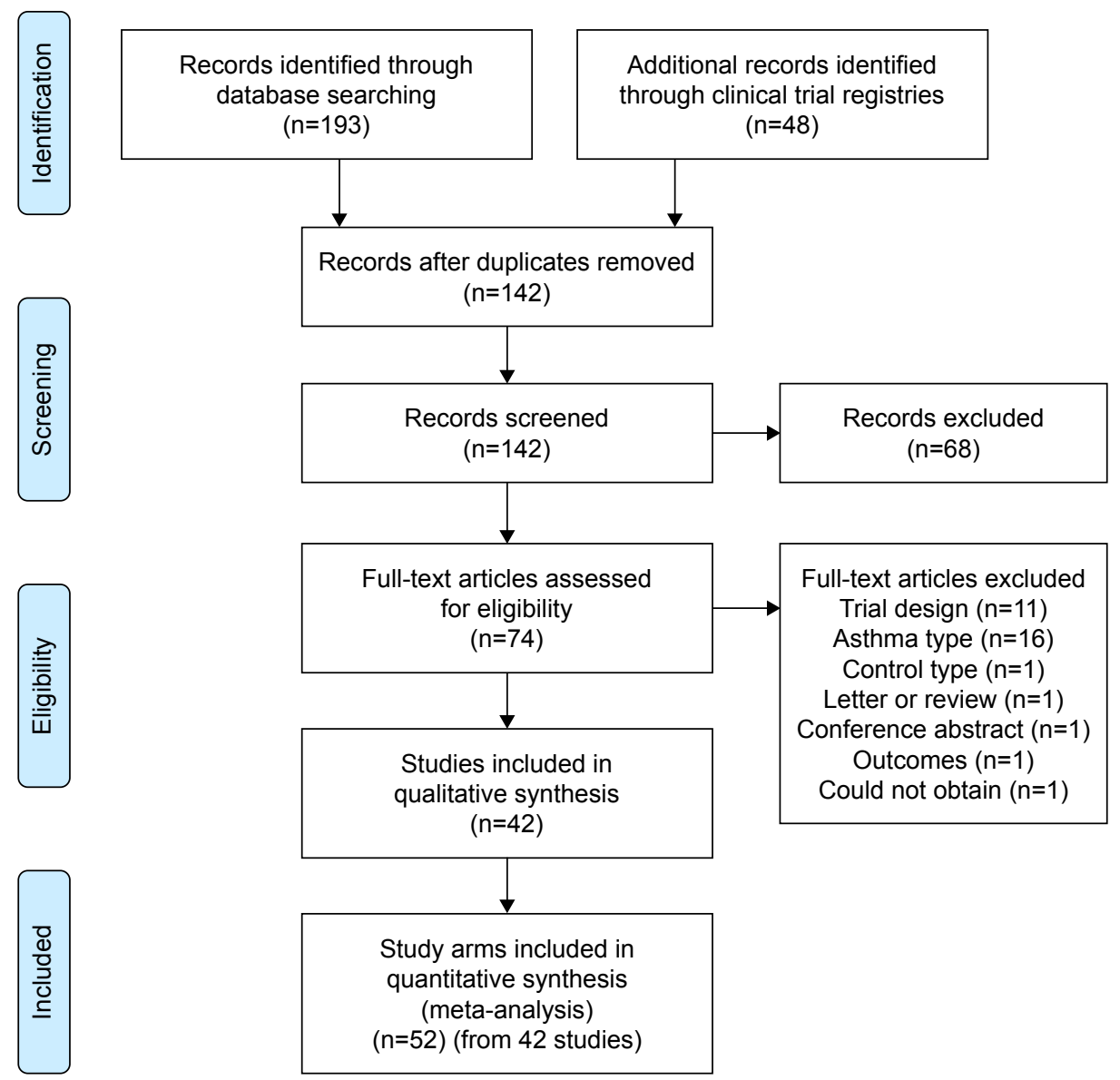

Figure I PRISMA flow diagram.

Notes: A total of 193 records were identified through database searching and other sources. After removal of duplicates, 142 records remained. Exclusion of 68 records at the title/abstract level resulted in 74 articles to be examined as full text. Of these, 32 full-text articles were excluded. Fifty-two study arms from 42 studies were included in the final analysis.

Abbreviation: PRISMA, Preferred Reporting Items for Systematic Reviews and Meta-Analyses.

\section{Other outcomes}

Other outcomes extracted were symptom scores, admission rate, duration of stay, use of rescue medication, and oxygen saturation (Figure 7). In almost every case, there were no significant differences between theophylline and control. The exception was the duration of hospital stay (Figure 7C), with theophylline reducing the duration of stay by 0.23 hours (14 minutes) (95\% CI: $-0.37,-0.08$ hours, $p=0.002)$.

\section{Subgroup analysis: background medication}

Theophylline was neither more nor less effective than control treatments for almost all outcomes. This was true whether the control was an active comparator like salbutamol, or a placebo. We questioned whether the regimen of medications given to patients before or during the studies ("background medication") was responsible for the perceived lack of additional efficacy of theophylline over placebo.

In order to investigate this question, we undertook a subgroup analysis of FEV1 by background medication
(Figure 8). In studies where the background medication was oxygen only or no additional medication other than the study drug, the control drugs performed better than theophylline $(p<0.00001)$. Conversely, where patients were given bronchodilators with or without steroids, there was no significant differences between theophylline and control. Removal of the two studies comparing theophylline with placebo did not change the outcome.

\section{Subgroup analysis: age of participants}

As mentioned earlier, approximately two-thirds of the studies were conducted in adults, with one-third in children. In order to determine whether children responded differently to theophylline compared with adults, we intended to undertake a subgroup meta-analysis of FEV1 and PEFR by the age of participants. Unfortunately, these outcomes were rarely reported in children, as younger people can have great difficulty performing the necessary tests. Instead, we did a subgroup meta-analysis of symptom scores by age (Figure 9). 


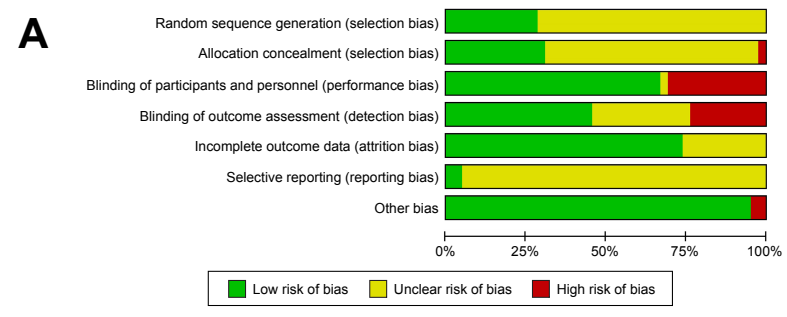

B

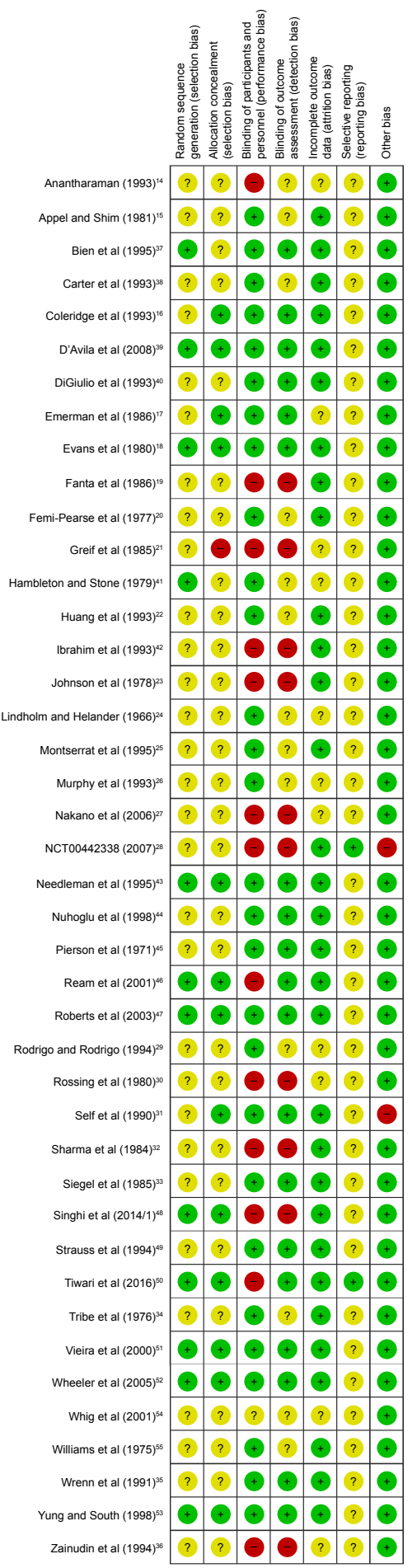

Figure 2 Assessment of study quality.

Notes: (A) Risk of bias graph. (B) Risk of bias summary. Each included study was assessed for selection bias, performance bias, detection bias, reporting bias, and other bias. Green: low risk of bias; Yellow: unclear risk of bias; Red: high risk of bias.
We found that there was no significant difference between adults and children in terms of symptoms $(p=0.38)$.

\section{Subgroup analysis: blinded vs unblinded studies}

In order to determine if blinding had any effect on the primary outcome (FEV1), we conducted a subgroup analysis of blinded vs unblinded studies. Studies that did not mention blinding were regarded as "unblinded". We found a slightly decreased efficacy for theophylline compared with controls in unblinded studies (Figure 10), although the difference between blinded and unblinded studies was not significant $(p=0.18)$. Removal of placebo-controlled trials from the analysis did not change the results (data not shown).

\section{Adverse events}

Fortunately, many studies reported on adverse events (Figure 11). Compared with placebo, IV theophylline caused more nausea, vomiting, and cardiovascular adverse events (such as palpitations and arrhythmias) (Figure 11A). There were no differences in abdominal pain, psychological side effects, headaches, seizures, or tremor. Compared with active comparators (Figure 11B), theophylline again caused more nausea and vomiting, but was not different from the active controls in terms of the frequency of psychological side effects, headaches, cardiovascular adverse events, tremor, CPK/CK elevation, or glucosuria/hyperglycemia.

\section{Publication bias}

In order to test for publication bias, we created funnel plots (Figure 12). The funnel plot for FEV1 (Figure 12A) did not show significant asymmetry. This was true also for PEFR (Figure 12B), symptom scores (Figure 12C), or heart rate (Figure 12D).

\section{Discussion}

Our study has comprehensively reviewed the combined evidence for the efficacy and safety of IV theophylline in acute asthma. We found that theophylline somewhat reduced the heart rate and duration of stay, and was not significantly worse than adrenaline, beta- 2 agonists, and leukotriene receptor antagonists. Furthermore, apart from an increase in nausea and vomiting, side effects from theophylline were not significantly different from other treatment regimes. That is, although theophylline was not clinically superior to other treatments, it was not significantly worse either.

However, of great importance was our subgroup analysis of FEV1 by the background medication given to patients (Figure 8 ). Where the patients were given no medication, 
A

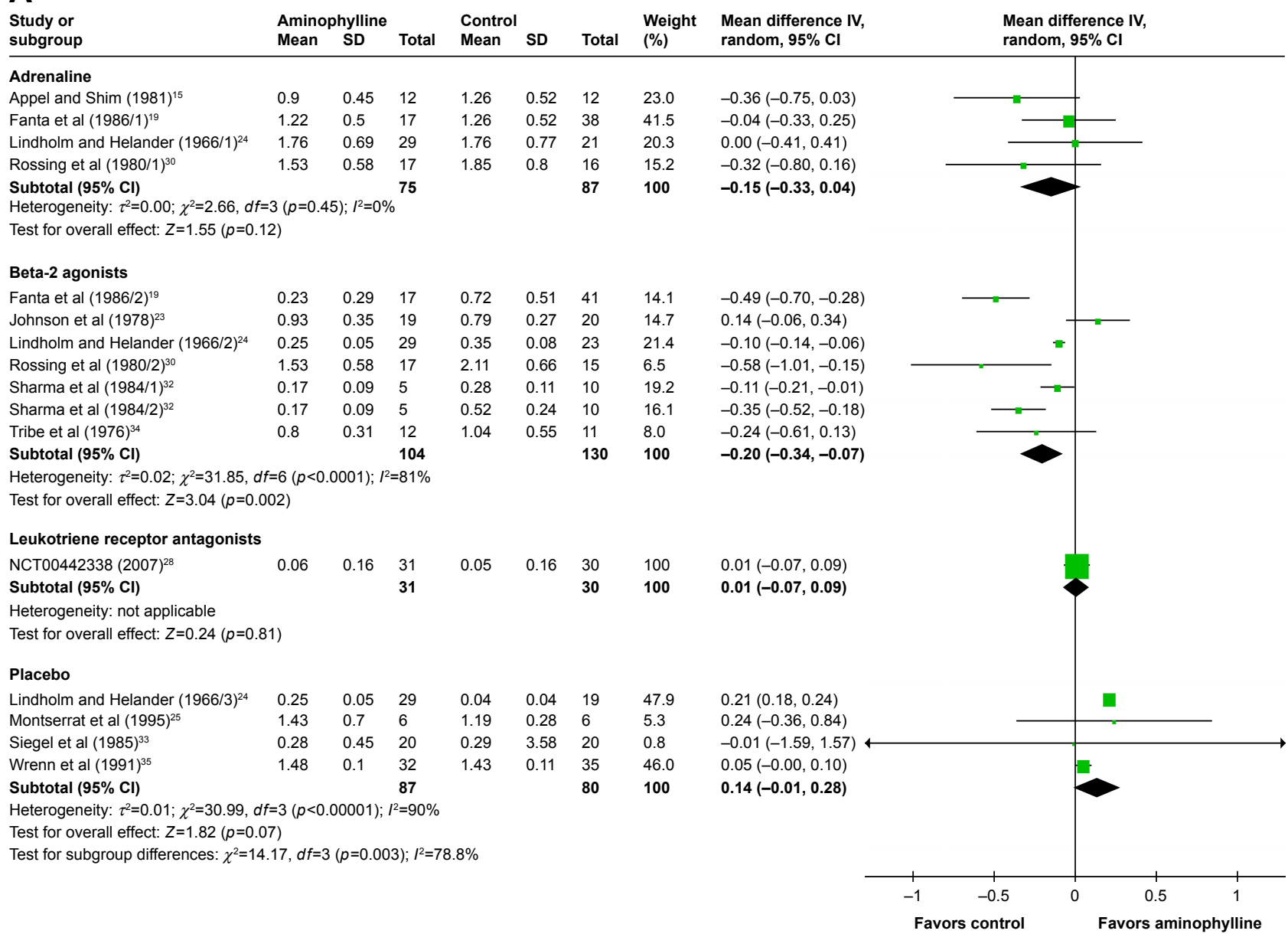

B

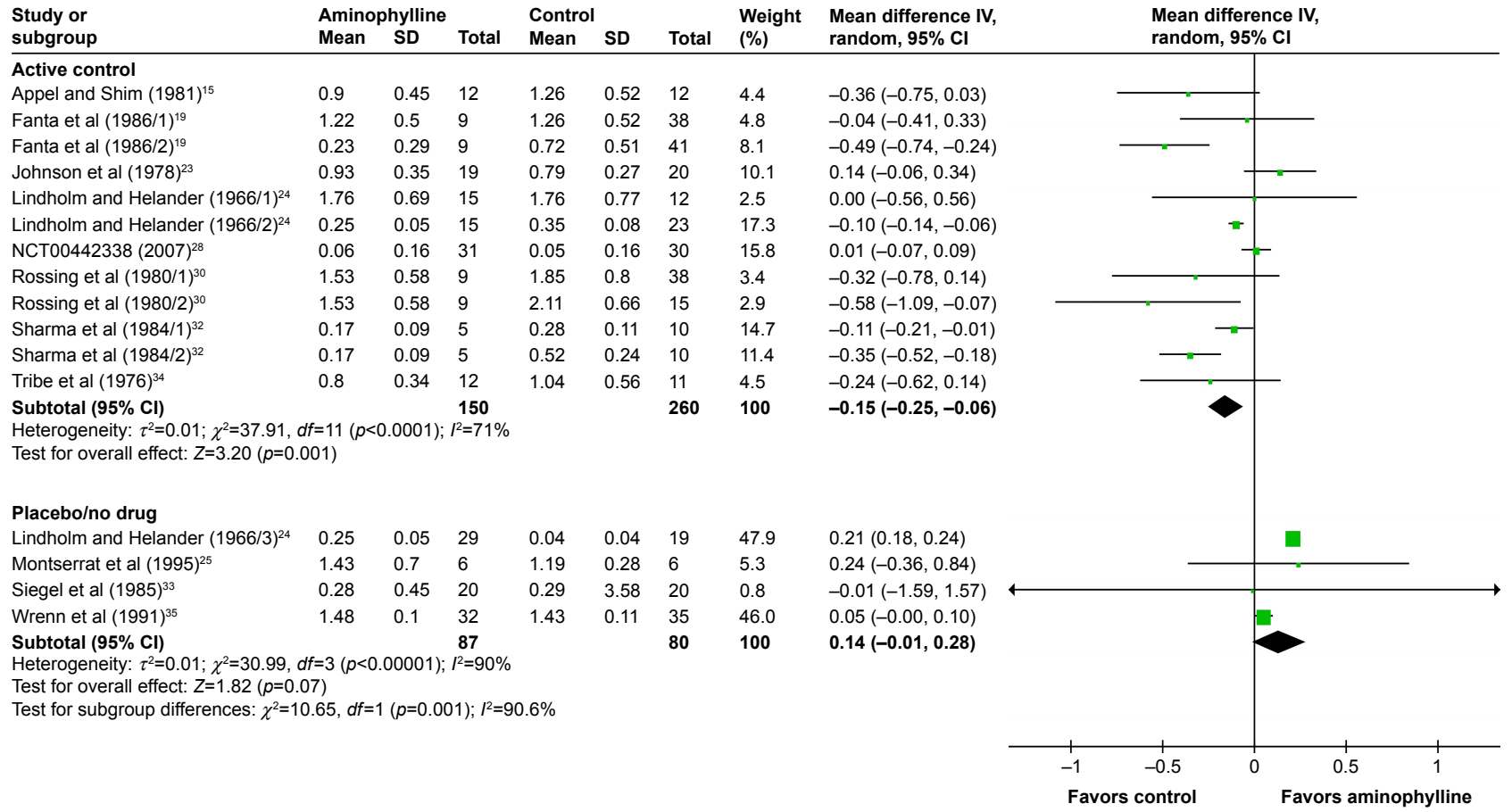

Figure 3 (Continued) 


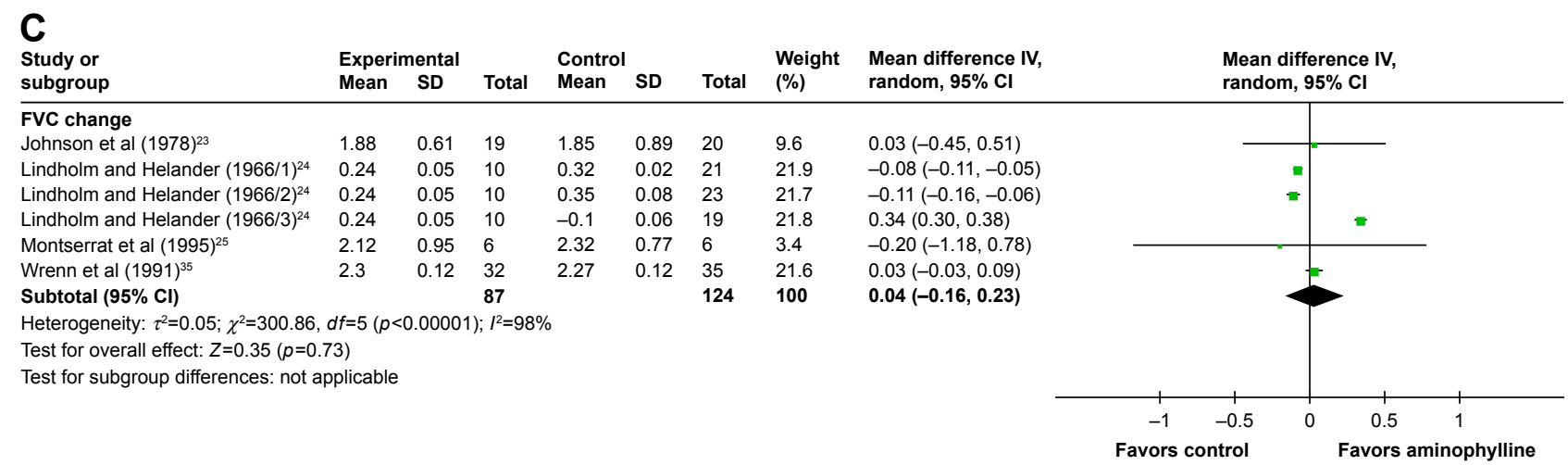

Figure 3 Meta-analysis of FEVI (A, B) or FVC (C).

Notes: (A) Subgroup meta-analysis of FEVI (in liters [L]) following intravenous theophylline by control type. Controls included adrenaline, beta-2 agonists, leukotriene receptor antagonists, and placebo/no drug. (B) Subgroup meta-analysis of FEVI (L) following intravenous theophylline by control type (pooled active control vs placebo/no drug). (C) Meta-analysis of FVC following intravenous theophylline, as measured in L. Data are given as the mean difference $(95 \% \mathrm{Cl})$.

A

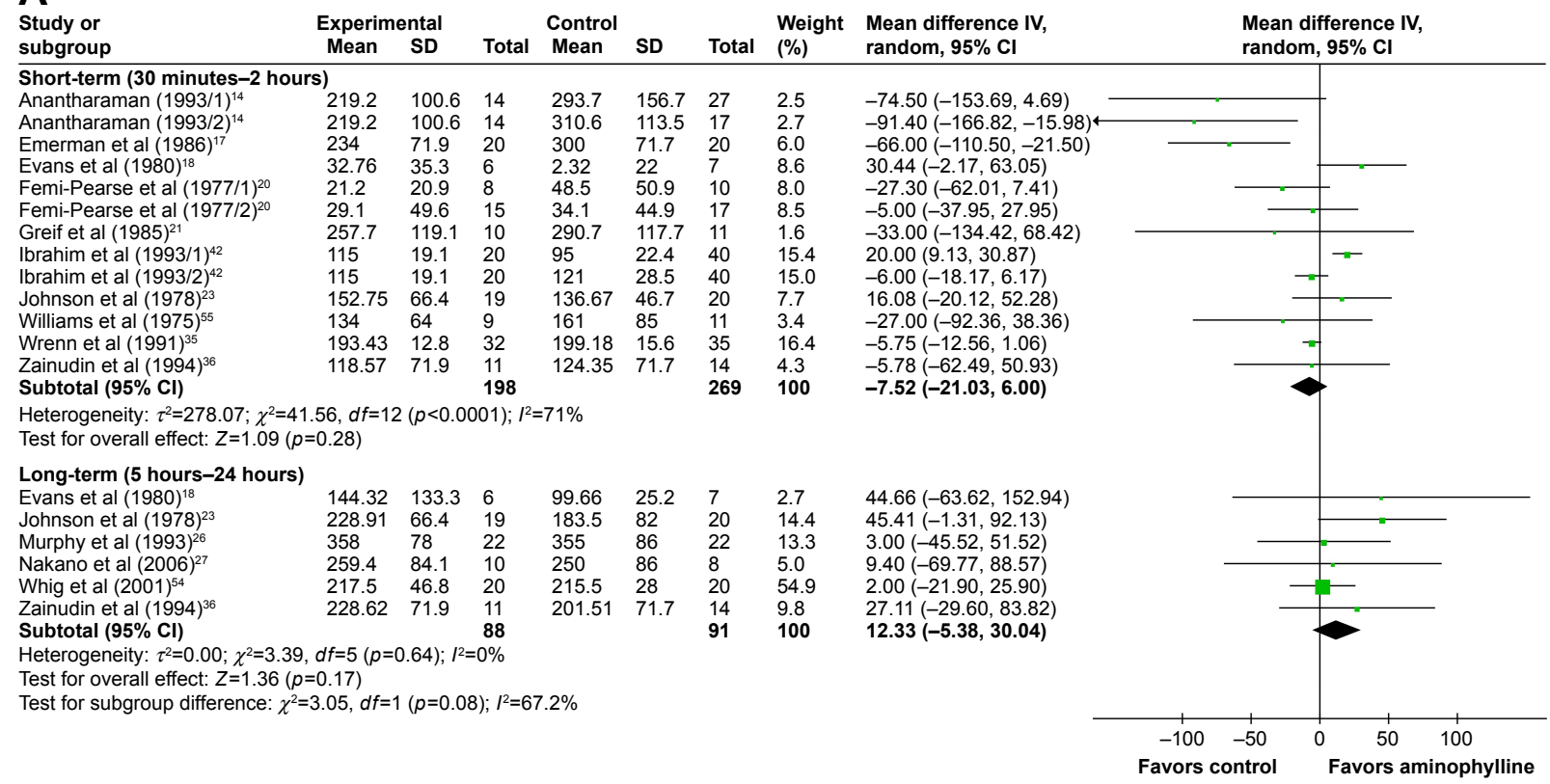

B

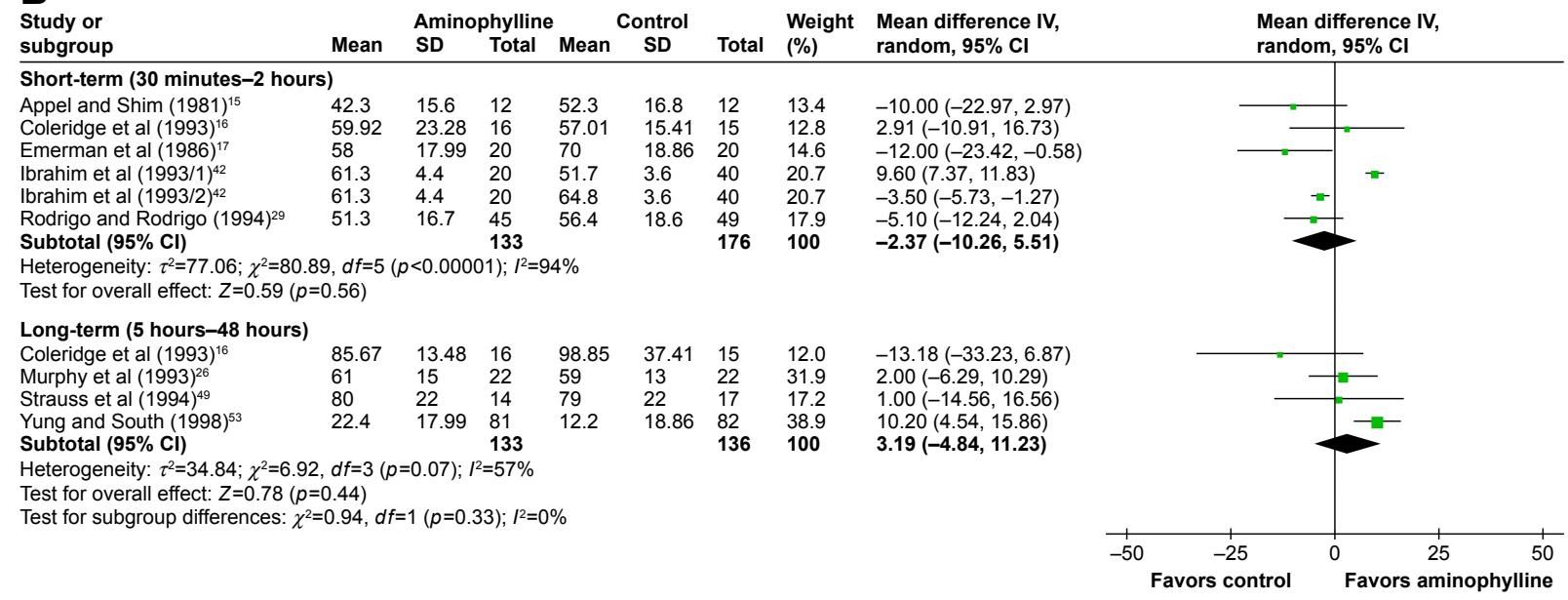

Figure 4 Subgroup meta-analysis of PEFR following intravenous theophylline by time post-infusion, as measured in liters (A) or as percent of predicted value (B).

Notes: Short-term follow-up was defined as 30 minutes-2 hours post-infusion. Long-term follow-up was defined as 5 hours- 36 hours post-infusion. Data are given as the mean difference $(95 \% \mathrm{Cl})$.

Abbreviation: PEFR, peak expiratory flow rate. 
A

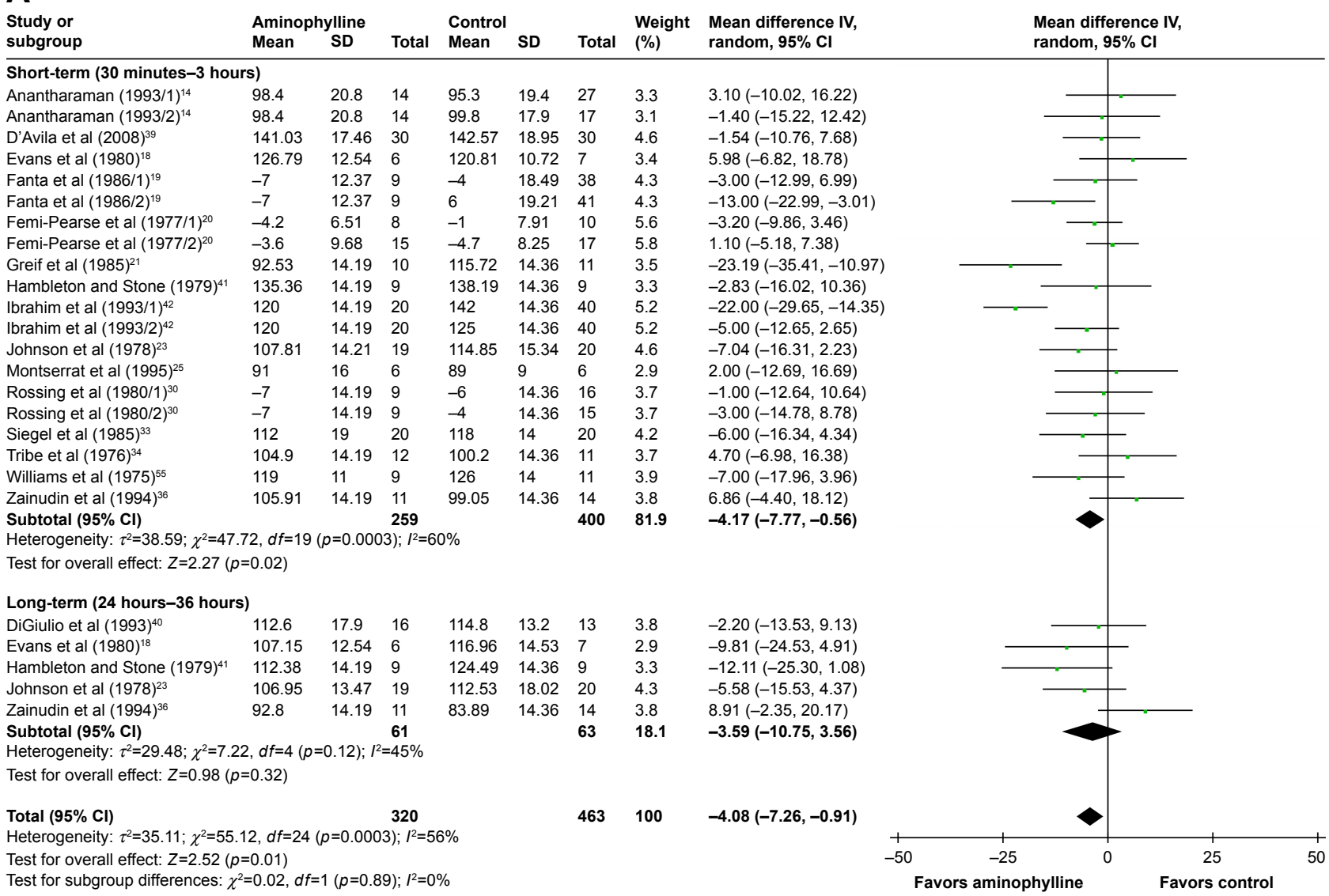

B

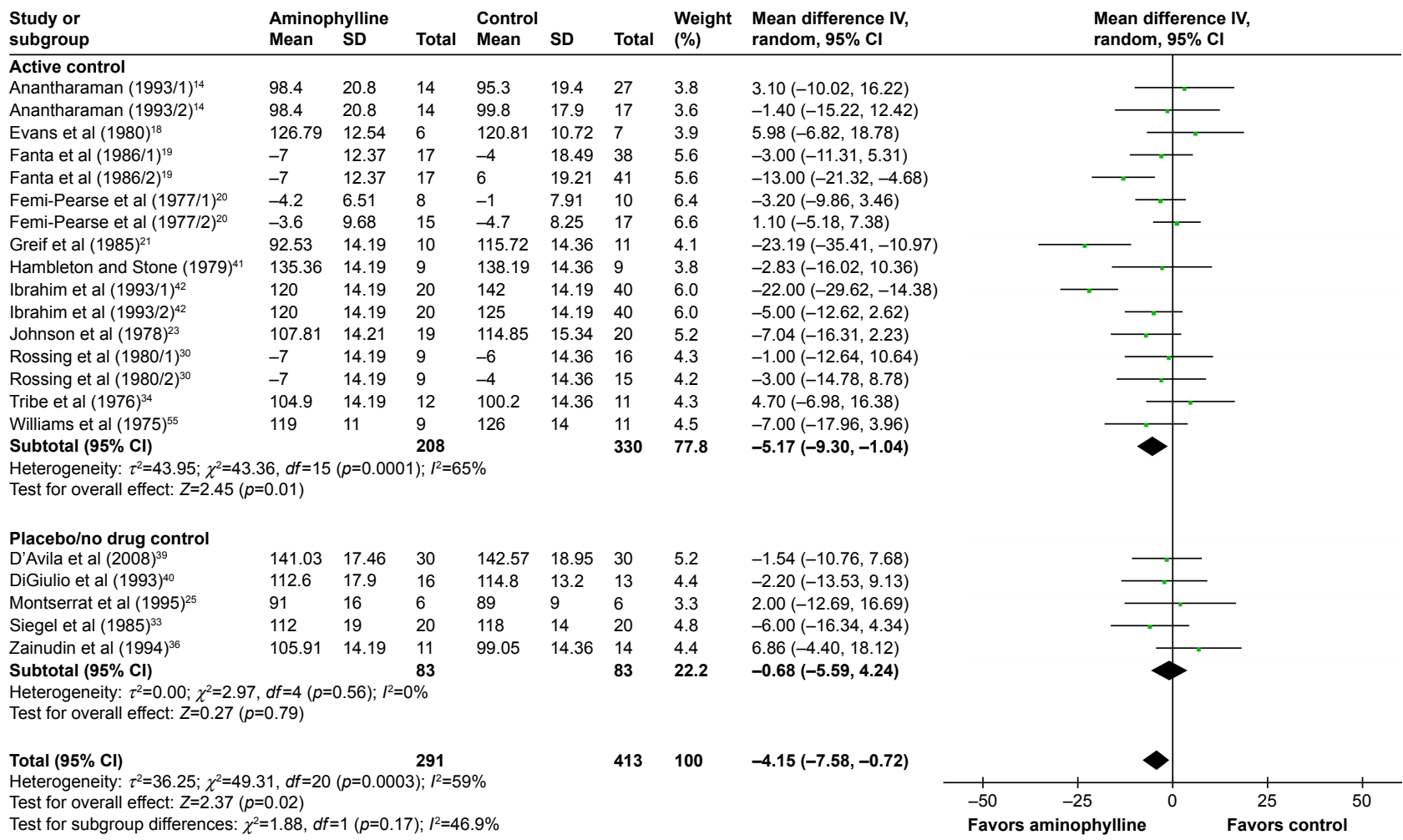

Figure 5 Subgroup meta-analysis of heart rate (beats per minute) following intravenous theophylline by time after infusion (A) and by type of control (B).

Notes: (A) Short-term follow-up was defined as 30 minutes 3 hours post-infusion. Long-term follow-up was defined as 24-36 hours post-infusion. (B) Active control was defined as administration of any drug with the aim of reducing the asthma exacerbation. Placebo was defined as a substance given that contains no active ingredient and is designed to maintain blinding of a clinical trial. Data are given as the mean difference $(95 \% \mathrm{Cl})$. 


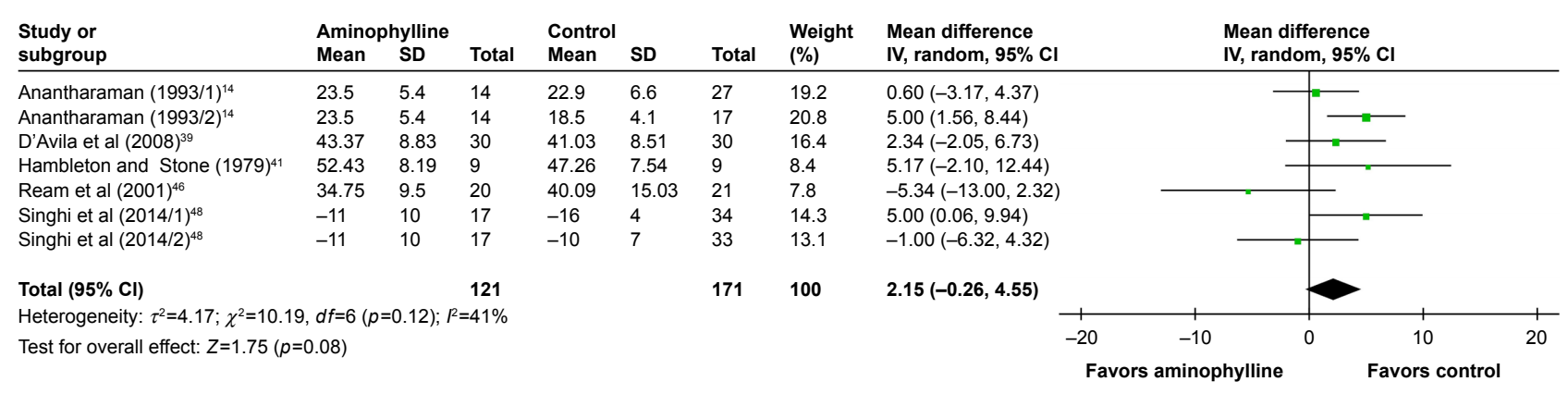

Figure 6 Meta-analysis of respiratory rate (breaths per minutes) following intravenous theophylline infusion.

Note: Data are given as the mean difference $(95 \% \mathrm{Cl})$.

A

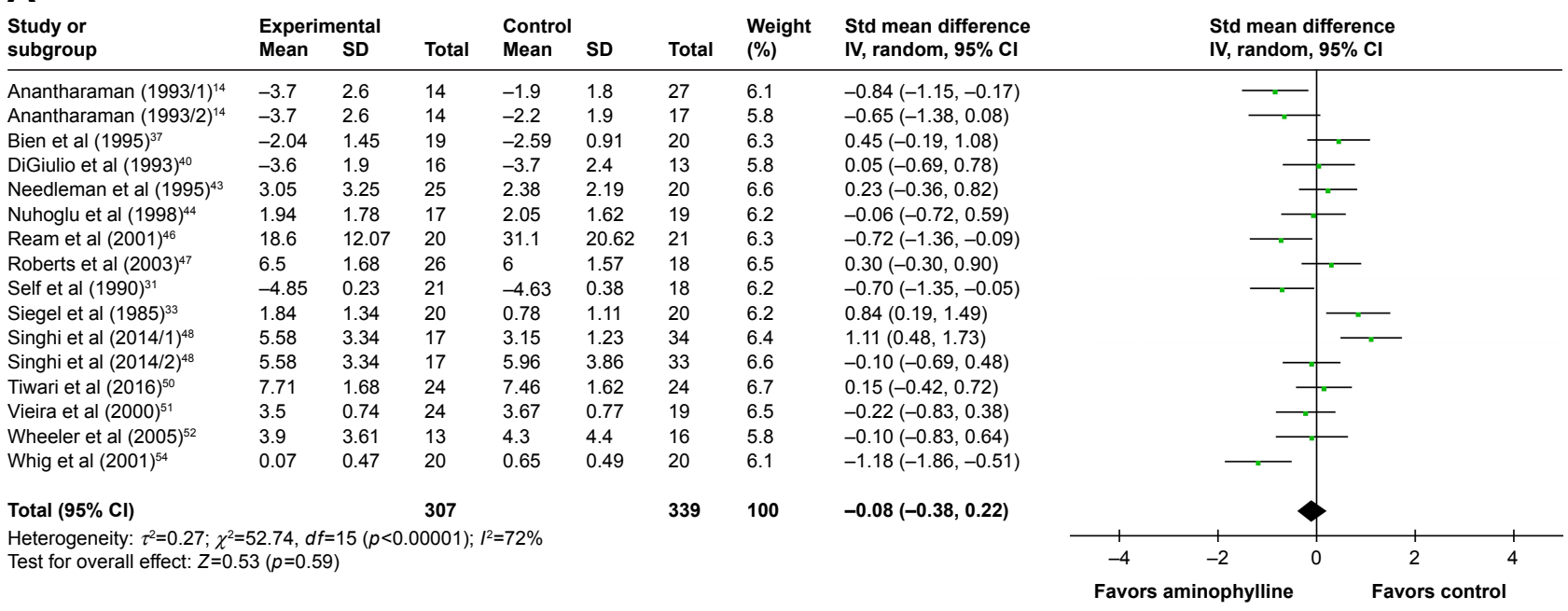

B

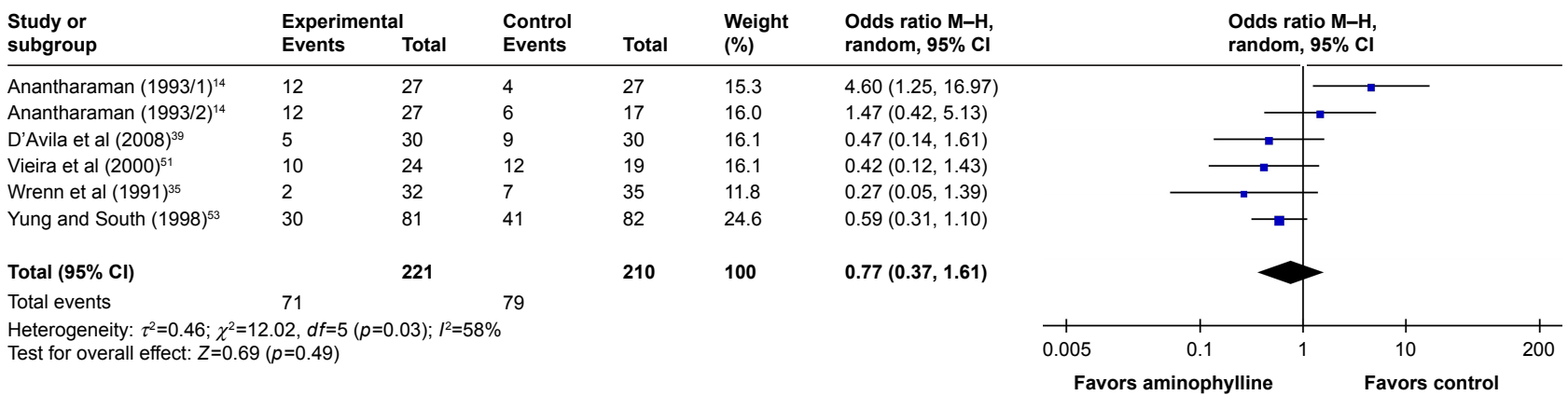

C

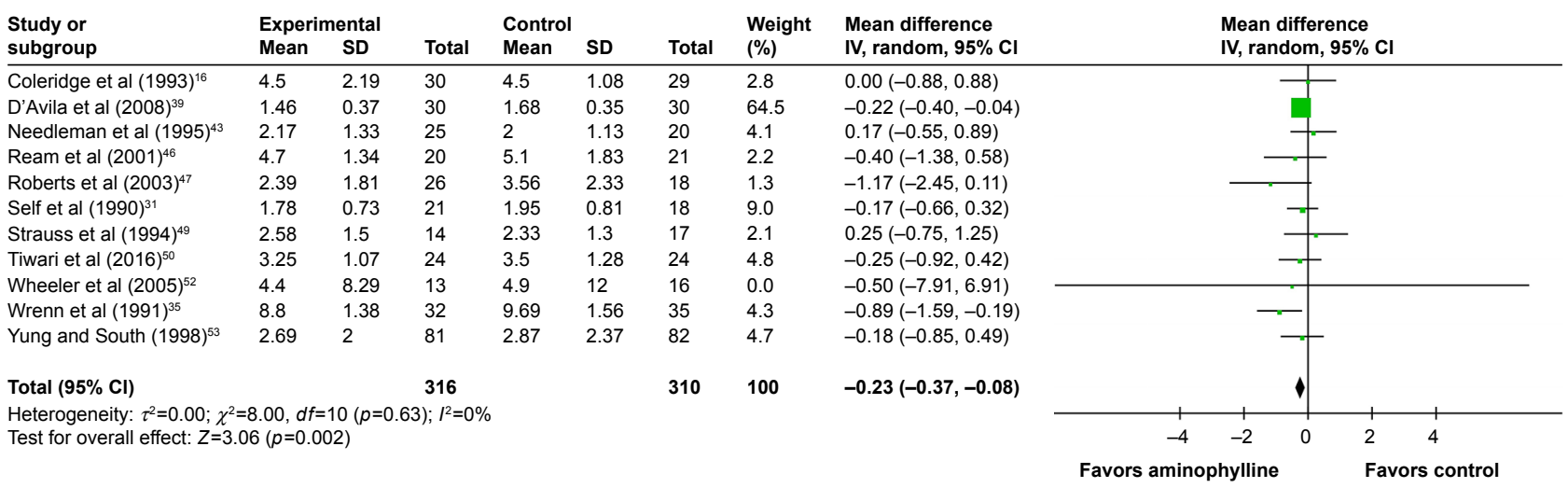

Figure 7 (Continued) 
D

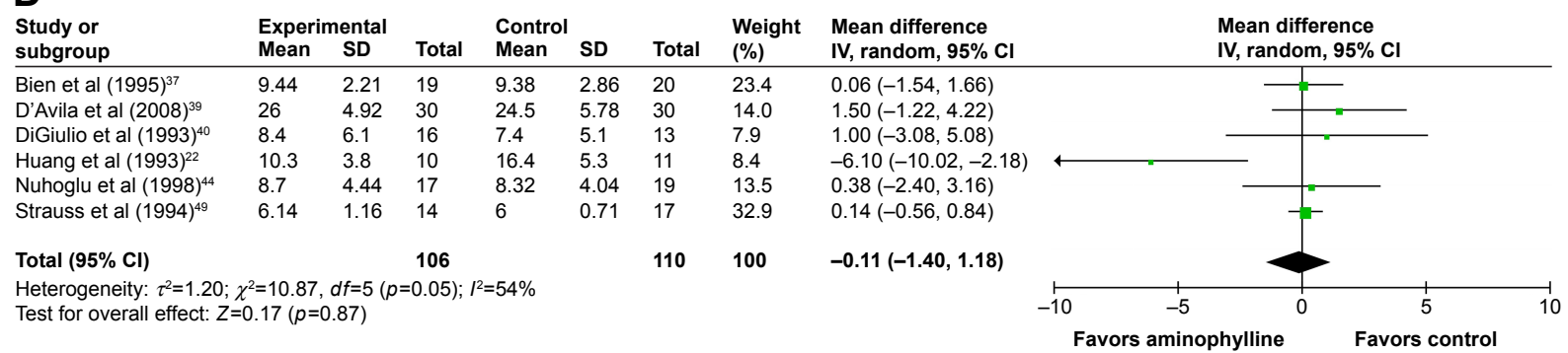

E

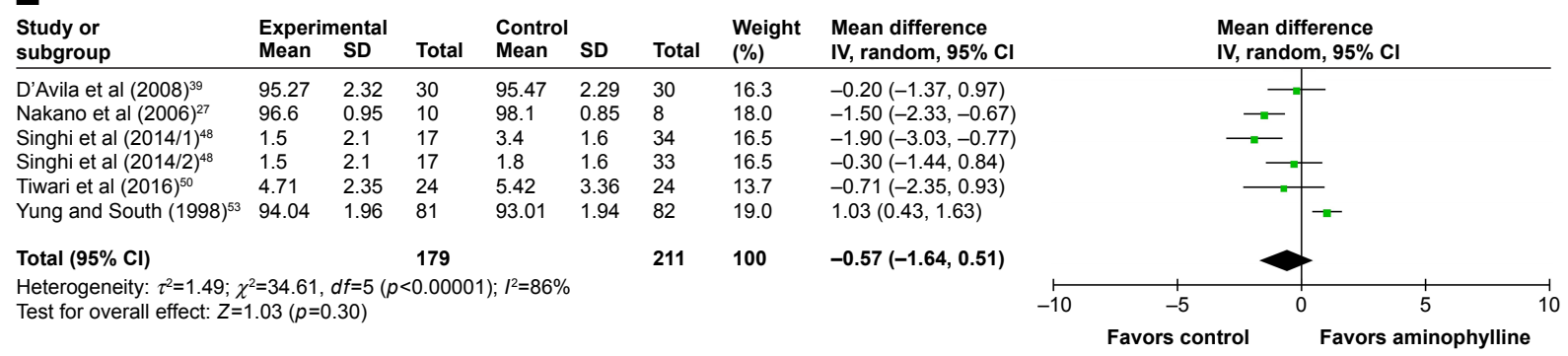

Figure 7 Meta-analysis of symptom scores (A), admission rates (B), duration of hospital stay (C), rescue medication use (D), and oxygen saturation (E) following intravenous theophylline.

Note: Data are given as standardized mean difference $(95 \% \mathrm{Cl})(\mathbf{A})$, odds ratios $(95 \% \mathrm{Cl})(\mathbf{B})$, or mean difference $(95 \% \mathrm{Cl})(\mathbf{C}-\mathbf{E})$.

or oxygen alone, theophylline was inferior to subcutaneous epinephrine ${ }^{15,19,30}$ and nebulized isoproterenol. ${ }^{19,30}$ However, in almost all circumstances, patients admitted to an emergency department for an acute asthma exacerbation are given nebulized beta- 2 agonists and IV corticosteroids. ${ }^{57-59}$ If these treatments fail, additional treatments are then considered.
Our data show that in the context of usual emergency department treatment, IV theophylline is at least as effective as montelukast ${ }^{28}$ and IV salbutamol. ${ }^{23,34}$

Existing studies on theophylline point to inconsistencies. For example, Neame et al attempted to determine if salbutamol or theophylline should be used for acute severe

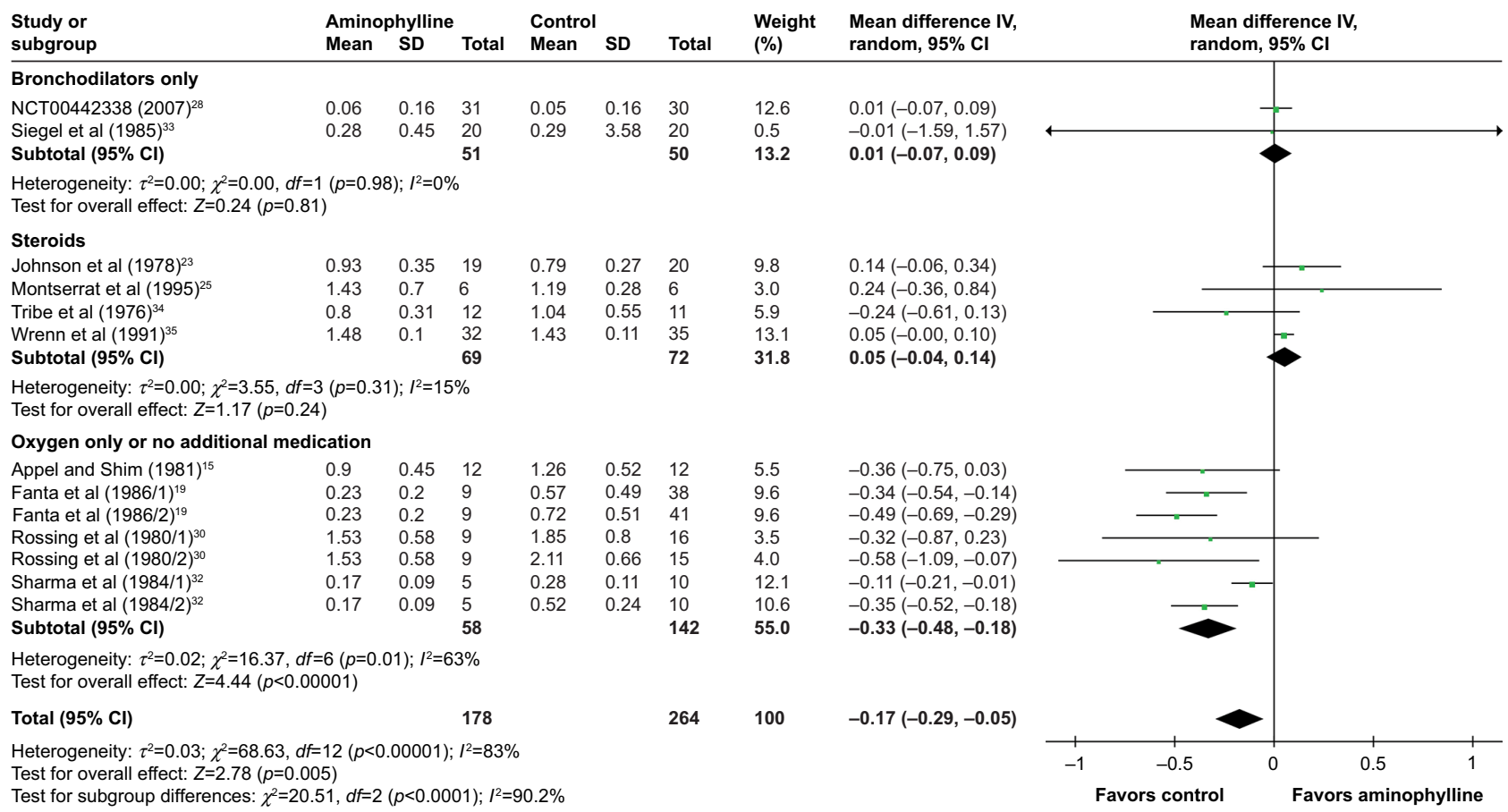

Figure 8 Subgroup meta-analysis of FEVI following intravenous theophylline by background medication.

Notes: Background medication is defined as the medication given to all participants, in addition to which theophylline or control was added. Subgroups are bronchodilators only, steroids with or without bronchodilators, and oxygen only or no additional medication. 


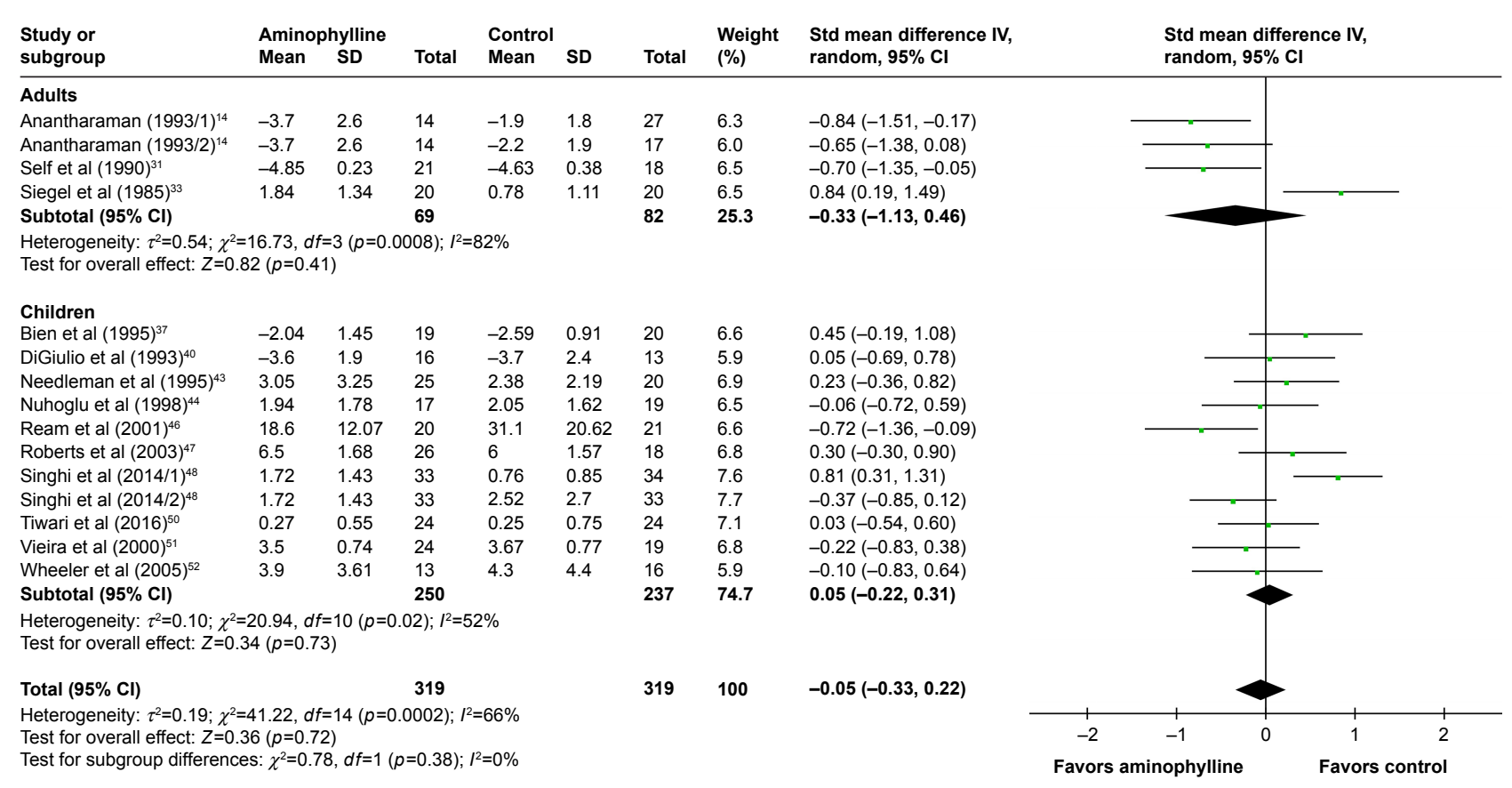

Figure 9 Subgroup meta-analysis of symptom scores following intravenous theophylline by age group.

Notes: Studies were grouped by the age of participants (children or adults). Studies with no stated age group or that did not enrol a particular age group were excluded from this analysis. Data are given as the mean difference $(95 \% \mathrm{Cl})$.

asthma in children. ${ }^{60}$ Despite a systematic search for articles, their qualitative analysis failed to draw any conclusions, due to "minimal and inconsistent" evidence.

A retrospective case-control study suggested that administration of theophylline increased hospital stay, compared with inhaled beta-2 agonists and corticosteroids. ${ }^{61}$
A meta-analysis by Mitra et al found that, in children, addition of theophylline to nebulized short-acting beta-2 agonists and systemic steroids resulted in better lung function in the first 6 hours of treatment. ${ }^{62}$ However, Mitra et al did not investigate the addition of other drugs to the same background therapy.

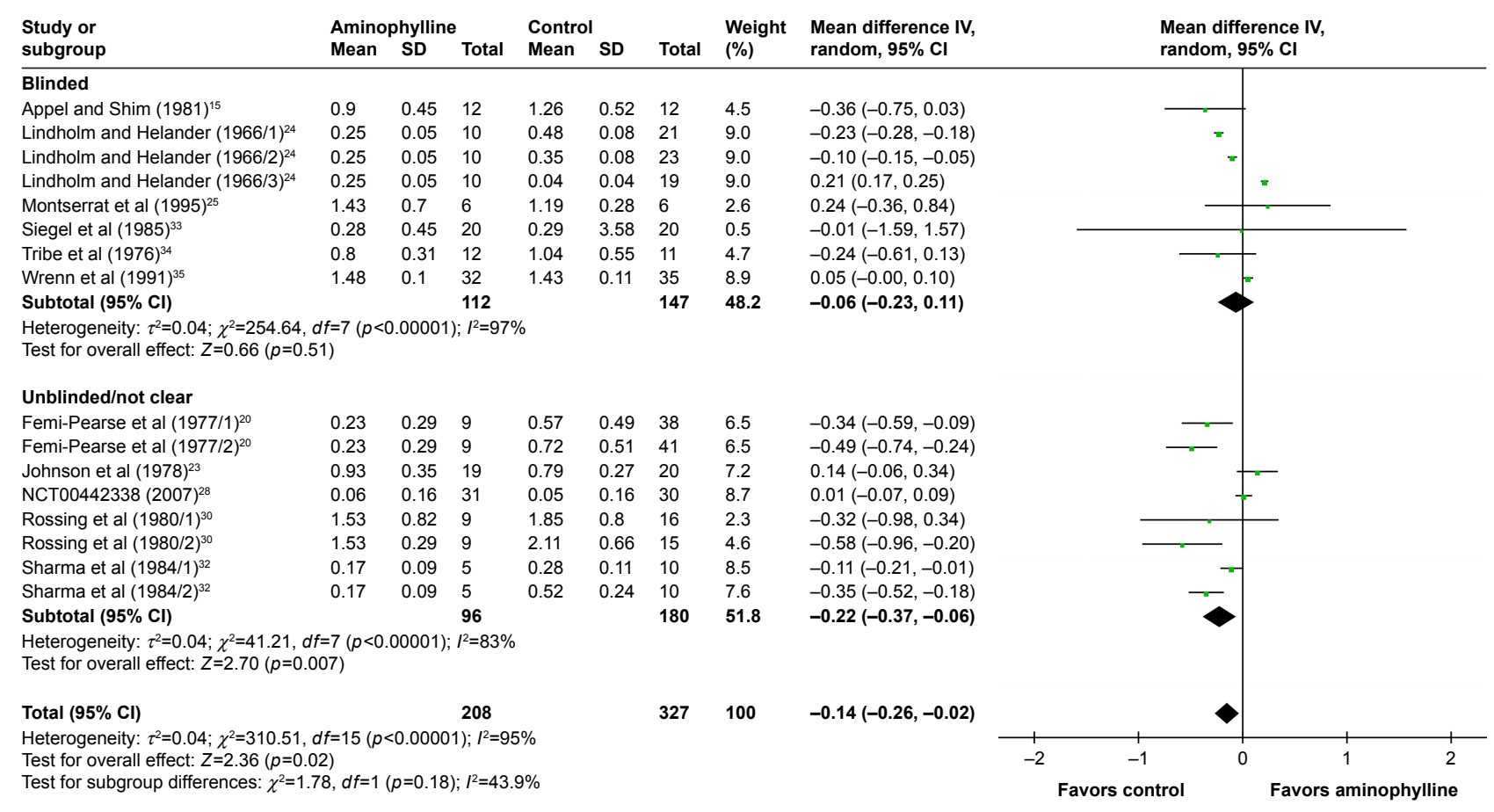

Figure 10 Subgroup meta-analysis of FEVI following intravenous theophylline by blinding of study participants.

Note: Data are given as the mean difference $(95 \% \mathrm{Cl})$. 


\section{A}

\begin{tabular}{|c|c|c|c|}
\hline Study or subgroup & $\begin{array}{l}\text { Experimental } \\
\text { Events }\end{array}$ & Total & $\begin{array}{l}\text { Contrc } \\
\text { Events }\end{array}$ \\
\hline \multicolumn{4}{|l|}{ Nausea } \\
\hline Bien et al $(1995)^{37}$ & 7 & 19 & 0 \\
\hline Huang et al $(1993)^{22}$ & 3 & 10 & 1 \\
\hline Ream et al $(2001)^{46}$ & 13 & 20 & 8 \\
\hline Rodrigo and Rodrigo (1994) ${ }^{29}$ & 17 & 45 & 3 \\
\hline Siegel et al $(1985)^{33}$ & 2 & 20 & 0 \\
\hline Strauss et al (1994) ${ }^{49}$ & 5 & 14 & 0 \\
\hline Whig et al $(2001)^{5^{\prime}}$ & 8 & 20 & 2 \\
\hline Wrenn et al (1991) $)^{35}$ & 3 & 32 & \\
\hline Yung and South $(1998)^{53}$ & 29 & 81 & 7 \\
\hline Zainudin et al (1994) $)^{36}$ & 3 & 11 & 0 \\
\hline Subtotal $(95 \% \mathrm{Cl})$ & & 272 & \\
\hline Total events & 90 & & 22 \\
\hline
\end{tabular}

Events

$\begin{array}{ll}\text { Total } & \text { Weigh } \\ & \\ 20 & 0.9 \\ 11 & 1.3 \\ 21 & 2.9 \\ 49 & 2.8 \\ 20 & 0.9 \\ 17 & 0.9 \\ 20 & 2.1 \\ 35 & 1.4 \\ 82 & 3.9 \\ 14 & 0.9 \\ \mathbf{2 8 9} & \mathbf{1 8 . 0} \\ & \\ \end{array}$

Weight (\%)

Odds ratio $\mathrm{M}-\mathrm{H}$

Odds ratio $\mathrm{M}-\mathrm{H}$,

Heterogeneity: $\tau^{2}=0.00 ; \chi^{2}=3.58, d f=9(p=0.92$
Test for overall effect: $Z=6.66(p<0.00001)$

Vomiting

Bien et al $(1995)^{37}$

DiGiulio et al $(1993)^{40}$

Murphy et al (1993)

Ream et al (2001)

Strauss et al (1994)

Whig et al (2001)

Yung and South (1998)

Subtotal $(95 \% \mathrm{Cl})$

Total events

Heterogeneity: $\tau^{2}=0.00 ; \chi^{2}=1.84, d f=7 \quad(p=0.97) ; l^{2}=0 \%$

Test for overall effect: $Z=6.15(p<0.00001)$

Abdominal pain

Ream et al (2001) ${ }^{46}$

Strauss et al (1994)

Subtotal $(95 \%$
Total events

Total events $\quad \begin{gathered}6 \\ \text { Heterogeneity: } \tau^{2}=0.85 ; \chi^{2}=1.54, d f=1 \\ (p=0.21) ; l^{2}=35 \%\end{gathered}$

Psychological

Bien et al $(1995)^{37}$

Ream et al $(2001)^{46}$

Rodrigo and Rodrigo (1994) ${ }^{29}$

Siegel et al (1985)

Whig et al $(2001)^{54}$
Yung and South $(1998)^{53}$

Yung and South (1998)

Zainudin et al (1994)

Total events

6
6
10
14
4
2
4
35

81
$(p=0.97) ; r^{2}=0 \%$
$01)$

19
16
22
20
20
14
20
81
212

3
2
2
7
1
0
1
8
24

$\begin{array}{ll}20 & 2.4 \\ 13 & 2.0 \\ 22 & 2.2 \\ 21 & 2.8 \\ 20 & 1.4 \\ 17 & 0.9 \\ 20 & 1.4 \\ 82 & 4.0 \\ \mathbf{2 1 5} & \mathbf{1 7 . 0}\end{array}$

20
14
34

$\begin{array}{ll}0.9 & 24.60(1.29,469.00) \\ 1.3 & 4.29(0.37,50.20) \\ 2.9 & 3.02(0.85,10.78) \\ 2.8 & 9.31(2.50,34.65) \\ 0.9 & 5.54(0.25,123.08) \\ 0.9 & 20.26(1.01,407.35) \\ 2.1 & 6.00(1.08,33.27) \\ 1.4 & 3.52(0.35,35.67) \\ 3.9 & 5.98(2.43,14.67) \\ 0.9 & 11.94(0.55,260.28) \\ 18.0 & 6.05(\mathbf{3 . 5 6}, \mathbf{1 0 . 2 8})\end{array}$

Heterogeneity: $\tau^{2}=0.00 \cdot \chi^{2}=4.54, d f=6\left(\begin{array}{c}48 \\ (p=0.60) ; l^{2}=0 \%\end{array}\right.$

Test for overall effect: $z=1.19(p=0.23)$

\section{Headache}

Murphy et al $(1993)^{26}$

Ream et al (2001) ${ }^{46}$

Rodrigo and Rodrigo (1994) $)^{29}$

Strauss et al (1994)

Whig et al $(2001)^{54}$
Yung and South $(1998)^{53}$

Yung and

Total events

Heterogeneity: $\tau^{2}=0.13 ; \chi^{2}=5.92, d f=5(p=0.31) ; l^{2}=15 \%$

$(24$

17.0

$2.62(0.55,12.48)$

$3.30(0.54,20.27)$

$8.33(1.56,44.64)$

$4.67(1.25,17.44)$

$7.00(0.31,158.83)$

$4.75(0.48,46.91)$

$7.04(3.00,16.49)$

$\mathbf{5 . 3 5}(\mathbf{3 . 1 4}, \mathbf{9 . 1 2})$

Cardiovascular (palpitation, arrhythmia)

Murphy et al $(1993)^{26}$

Rodrigo and Rodrigo (1994) ${ }^{29}$

Siegel et al (1985)

Whig et al (2001)

Wrenn et al (1991) 35

Zainudin et al (1994)

Total events
Heterogeneity:
$\tau^{2}=0.00 ; \chi^{2}=4.49, d f=5(p=0.48) ; l^{2}=0 \%$

Heterogeneity: $\tau^{2}=0.00 ; \chi^{2}=4.49, d f=5$

Seizure

Wrenn et al (1991) $)^{35}$

Yung and South (1998) $)^{53}$

Subtotal $(95 \% \mathrm{Cl})$

Total events
Heterogeneity: $\tau^{2}=0.00 ; \chi^{2}=0.23, d f=1(p=0.63) ; l^{2}=0 \%$

\begin{tabular}{l} 
Test for overall effect: $Z=0.40 \quad(p=0.69)$ \\
\hline
\end{tabular}

DiGiulio et al (1993)40

DiGiulio et al (1993)

Ream et al (2001)

Rodrigo and Rodrigo (1994) ${ }^{29}$

Siegel et al (1985)

Wrenn et al (1991) ${ }^{35}$

Yung and South (1998)

Zainudin et al (1994)

Subtotal $(95 \% \mathrm{Cl})$

Total events 83

Heterogeneity: $\tau^{2}=0.94 ; \chi^{2}=22.11, d f=7(p$

Test for overall effect

Total $(95 \% \mathrm{Cl})$
Total events
Heterogeneity: $\tau^{2}=0.45 ; \chi^{2}=84.59, d f=48(p=0.0009) ; l^{2}=43 \%$

20
4
4

$\begin{array}{ll}21 & 2.5 \\ 17 & 0.9 \\ \mathbf{3 8} & \mathbf{3 . 4}\end{array}$

$0.80(0.18,3.54)$

$7.00(0.31,158.83)$
$\mathbf{1 . 5 2}(\mathbf{0 . 2 1}, \mathbf{1 0 . 7 9 )}$

Test for subgroup differences: $\chi^{2}=29.17, d f=7(p=0.0001) ; l^{2}=76.0 \%$

19
20
45
20
20
32
81
$\mathbf{2 3 7}$

$\begin{array}{ll}21 & 2.7 \\ 9 & 27 \\ 20 & 12 \\ 20 & 1.4 \\ 35 & 2.2 \\ 247 & 4.4\end{array}$

$\begin{array}{ll}2.1 & 4.15(0.72,23.95) \\ 2.7 & 0.83(0.21,3.33) \\ 2.7 & 0.86(0.22,3.42) \\ 1.2 & 2.11(0.18,25.35) \\ 1.4 & 6.33(0.67,60.16) \\ 2.2 & 1.10(0.21,5.90) \\ .4 & 1.23(0.61,2.47) \\ 6.8 & \mathbf{1 . 3 5}(\mathbf{0 . 8 2}, \mathbf{2 . 2 0})\end{array}$

$\begin{array}{ll}22 & 1 \\ 20 & 3 \\ 45 & 3 \\ 14 & 0 \\ 20 & 1 \\ 81 & 15 \\ \mathbf{2 0 2} & \end{array}$

$\begin{array}{ll}22 & 0.8 \\ 21 & 2.1 \\ 49 & 2.6 \\ 17 & 0.9 \\ 20 & 1.5 \\ 82 & 4.2 \\ \mathbf{2 1 1} & \mathbf{1 2 . 0}\end{array}$

$0.32(0.01,8.25)$
$1.06(0.19,5.99)$

$1.06(0.19,5.99)$
$2.82(0.68,11.68)$

$7.00(0.31,158.83)$

$8.14(0.88,75.48)$

$\mathbf{1 . 6 0}(\mathbf{0 . 7 8}, \mathbf{3 . 3 0})$

$4.38(0.99,19.36)$

$3.64(1.07,12.43)$

$1.00(0.06,17.18)$
$3.15(0.12,82.16)$

$3.15(0.12,82.16)$
$1.10(0.15,8.30)$

$1.10(0.15,8.30)$
$22.75(2.11,244.87)$

$3.52(1.66,7.49)$

Figure I I (Continued) 


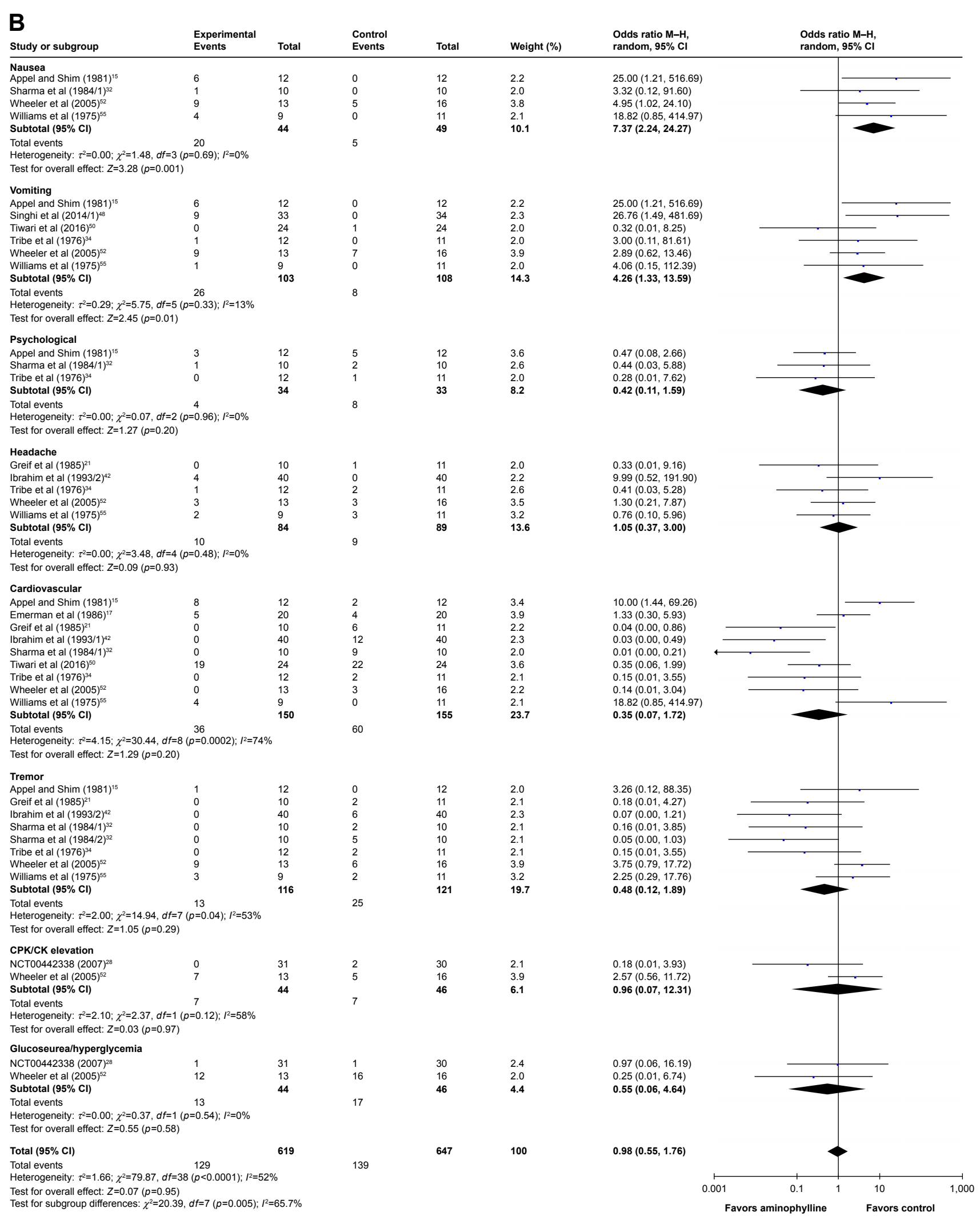

Figure II Subgroup meta-analysis of adverse events in placebo-controlled trials (A) or active comparator trials (B).

Note: Data are given as odds ratios $(95 \% \mathrm{Cl})$. 
A
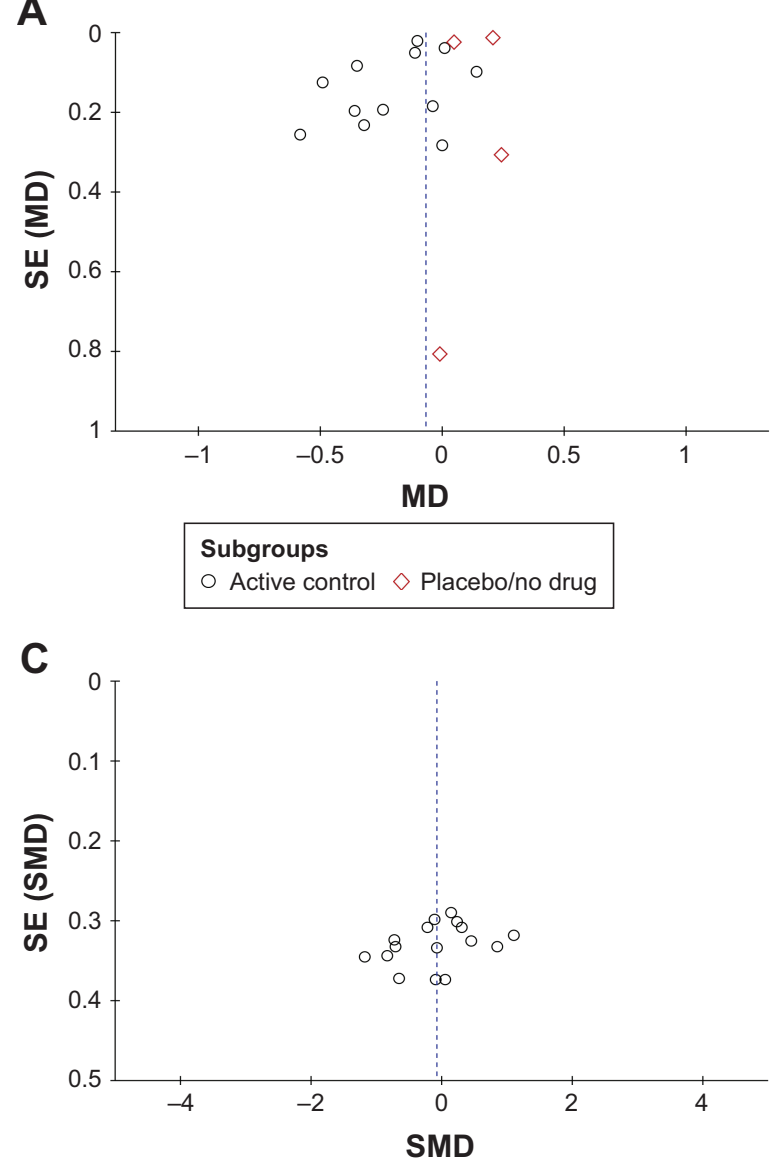

B

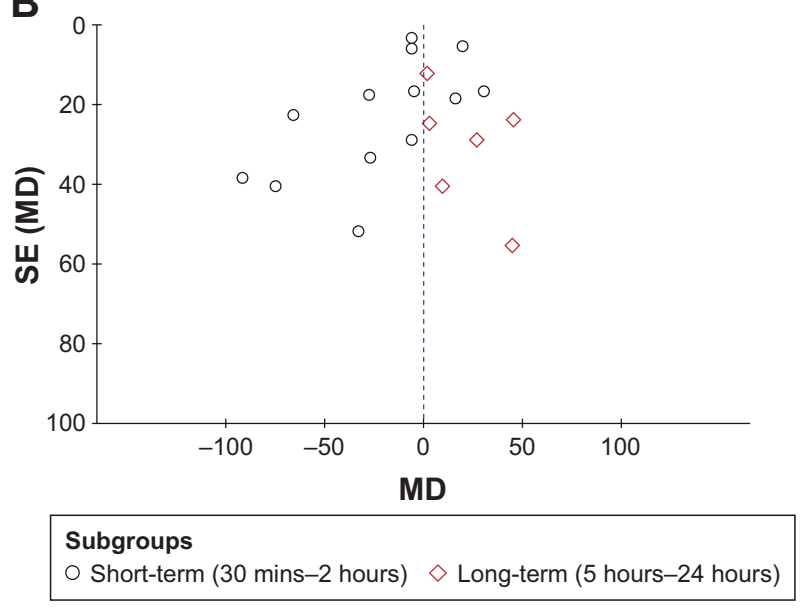

D

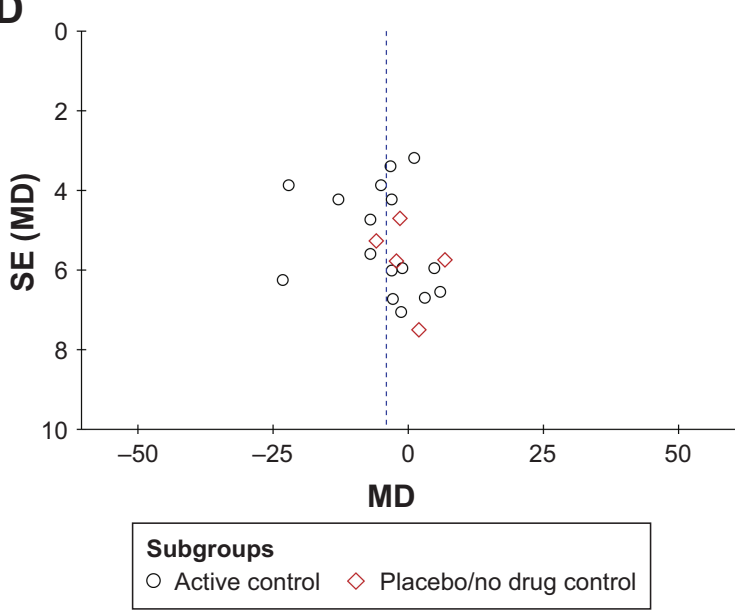

Figure 12 Funnel plot analysis of FEVI (A), PEFR (B), symptom score (C), and heart rate (D).

Abbreviations: SE, standard error; MD, mean difference; PEFR, peak expiratory flow rate; SMD, standardized mean difference.

In contrast, a more recent meta-analysis analyzed trials directly comparing IV beta-2 agonists with IV theophylline in the treatment of acute asthma. ${ }^{63}$ In this meta-analysis, Travers et al found no significant differences between IV beta-2 agonists and IV theophylline added to normal treatment in terms of hospital stay, PEFR, FEV1, heart rate, or clinical failure. In addition, Nair et al found that adding IV theophylline to inhaled beta-2 agonists did not provide additional benefit in adults with acute asthma ${ }^{64}$ None of these meta-analyses specifically investigated the role of background medication in the efficacy of theophylline, compared with other additional medications.

Recent data from the UK suggest that, at least in children, theophylline was the third most commonly administered drug in an acute setting, after salbutamol and magnesium sulfate.$^{65}$ However, different drugs, especially new, branded formulations of drugs, may differ in cost by a large degree. Indeed, a 2005 study included hospital cost in their analysis. ${ }^{52}$ They found that treating their patients with theophylline was as effective as terbutaline, and the total treatment costs were less than a tenth of those with terbutaline.

\section{Limitations of this analysis}

We were fortunate to find a significant body of evidence testing the efficacy of theophylline. However, because asthma outcomes can be measured in a large number of different ways, we were limited in the investigations we could carry out. For example, we had planned to do meta-regression, but we felt there were insufficient studies in any one outcome to create a meaningful interpretation of the data. ${ }^{8}$

\section{Conclusion}

Our data show that IV theophylline is superior to other treatments with regard to heart rate and duration of hospital stay, and equal to other treatments for almost all our other reported 
outcomes. Given the very low cost and similar safety profile of theophylline, it must surely be considered a cost-effective treatment for acute asthma exacerbations, especially for developing countries with restricted health budgets.

\section{Acknowledgment}

This study was supported by The Xinjiang Uygur Autonomous Region Natural Science Foundation Funded Projects (number: 2017D01C251).

\section{Author contributions}

GM developed and designed the concept for the systematic review and meta-analysis; she wrote the initial draft, did interpretation of the analyzed results, and finalized the manuscript. HZ, JL, NT, and LR did literature search, data collection, extraction, and analysis. GM, HZ, JL, NT, and LR wrote different sections of the manuscript. GM did the critical revision of the intellectual content of the article. All authors read and approved the final version of the manuscript.

\section{Disclosure}

The authors report no conflicts of interest in this work.

\section{References}

1. To T, Stanojevic S, Moores G, et al. Global asthma prevalence in adults: findings from the cross-sectional world health survey. BMC Public Health. 2012;12:204.

2. Global Asthma Network. The global asthma report 2014. Auckland, New Zealand: Global Asthma Network; 2014.

3. Lundbäck B, Backman H, Lötvall J, Rönmark E. Is asthma prevalence still increasing? Expert Rev Respir Med. 2016;10:39-51.

4. WHO. Global surveillance, prevention and control of chronic respiratory diseases: a comprehensive approach [Internet]. WHO [cited May 31, 2017]. Available from: http://www.who.int/gard/publications/ GARD_Manual/en/. Accessed May 31, 2017.

5. List of Asthma Medications (62 Compared) [Internet]. Drugs.com [cited May 31, 2017]. Available from: https://www.drugs.com/condition/ asthma.html. Accessed May 31, 2017.

6. Theophylline T1633 [Internet]. Sigma-Aldrich [cited May 31, 2017]. Available from: http://www.sigmaaldrich.com/catalog/product/ sigma/t1633. Accessed May 31, 2017.

7. Pubchem. aminophylline $\mid \mathrm{C} 16 \mathrm{H} 24 \mathrm{~N} 10 \mathrm{O} 4$ - PubChem [Internet] [cited May 31, 2017]. Available from: https://pubchem.ncbi.nlm.nih.gov/ compound/aminophylline. Accessed May 31, 2017.

8. Higgins JP, Greeen S. Cochrane Handbook for Systematic Reviews of Interventions. The Cochrane Collaboration; 2011.

9. Thomas J, Brunton J, Graziosi S. EPPI-Reviewer 4: Software for Research Synthesis. EPPI-Centre Software. London: Social Science Research Unit, UCL Institute of Education; 2010.

10. Higgins JPT, Altman DG, Gøtzsche PC, et al. The Cochrane Collaboration's tool for assessing risk of bias in randomised trials. BMJ. 2011; 343:d5928.

11. Rohatgi A. WebPlotDigitizer [Internet]. Austin, Texas, USA; 2017. Available from: http://arohatgi.info/WebPlotDigitizer

12. The Nordic Cochrane Centre. Review Manager (RevMan). The Cochrane Collaboration. Copenhagen; 2014.

13. Mantel N, Haenszel W. Statistical aspects of the analysis of data from retrospective studies of disease. J Natl Cancer Inst. 1959;22:719-748.
14. Anantharaman V. Therapeutic regimes for acute bronchial asthma. Singap Med J. 1993;34:534-537.

15. Appel D, Shim C. Comparative effect of epinephrine and aminophylline in the treatment of asthma. Lung. 1981;159:243-254.

16. Coleridge J, Cameron P, Epstein J, Teichtahl H. Intravenous aminophylline confers no benefit in acute asthma treated with intravenous steroids and inhaled bronchodilators. Aust N Z J Med. 1993;23: 348-354.

17. Emerman CL, Crafford WA, Vrobel TR. Ventricular arrhythmias during treatment for acute asthma. Ann Emerg Med. 1986;15:699-702.

18. Evans WV, Monie RD, Crimmins J, Seaton A. Aminophylline, salbutamol and combined intravenous infusions in acute severe asthma. $\mathrm{Br} J$ Chest. 1980;74:385-389.

19. Fanta CH, Rossing TH, McFadden ER Jr. Treatment of acute asthma. Is combination therapy with sympathomimetics and methylxanthines indicated? Am J Med. 1986;80:5-10.

20. Femi-Pearse D, George WO, Ilechukwu ST, Elegbeleye OO, Afonja AO. Comparison of intravenous aminophylline and salbutamol in severe asthma. Br Med J. 1977;1:491.

21. Greif J, Markovitz L, Topilsky M. Comparison of intravenous salbutamol (albuterol) and aminophylline in the treatment of acute asthmatic attacks. Ann Allergy. 1985;55:504-506.

22. Huang D, O'Brien RG, Harman E, et al. Does aminophylline benefit adults admitted to the hospital for an acute exacerbation of asthma? Ann Intern Med. 1993;119:1155-1160.

23. Johnson AJ, Spiro SG, Pidgeon J, Bateman S, Clarke SW. Intravenous infusion of salbutamol in severe acute asthma. Br Med J. 1978; 1:1013-1015.

24. Lindholm B, Helander E. The effect on the pulmonary ventilation of different theophylline derivatives compared to adrenaline and isoprenaline. Acta Allergol. 1966;21:299-306.

25. Montserrat JM, Barbera JA, Viegas C, Roca J, Rodriguez-Roisin R. Gas exchange response to intravenous aminophylline in patients with a severe exacerbation of asthma. Eur Respir J. 1995;8:28-33.

26. Murphy DG, McDermott MF, Rydman RJ, Sloan EP, Zalenski RJ. Aminophylline in the treatment of acute asthma when beta 2-adrenergics and steroids are provided. Arch Intern Med. 1993;153:1784-1788.

27. Nakano J, Yano T, Yamamura K, et al. Aminophilline suppress the release of chemical mediators in treatment of acute asthma. Respir Med. 2006;100:542-550.

28. NCT00442338. Study of MK0476 in Adult Patients With Acute Asthma (0476-334). amp, Dohme Corp, editors. 2007; Available from: http:// clinicaltrials.gov/show/NCT00442338. Accessed February 12, 2017.

29. Rodrigo C, Rodrigo G. Treatment of acute asthma. Lack of therapeutic benefit and increase of the toxicity from aminophylline given in addition to high doses of salbutamol delivered by metered-dose inhaler with a spacer. Chest. 1994;106:1071-1076.

30. Rossing TH, Fanta CH, Goldstein DH, Snapper JR, McFadden ER Jr. Emergency therapy of asthma: comparison of the acute effects of parenteral and inhaled sympathomimetics and infused aminophylline. Am Rev Respir Dis. 1980;122:365-371.

31. Self TH, Abou-Shala N, Burns R, et al. Inhaled albuterol and oral prednisone therapy in hospitalized adult asthmatics. Does aminophylline add any benefit? Chest. 1990;98:1317-1321.

32. Sharma TN, Gupta RB, Gupta PR, Purohit SD. Comparison of intravenous aminophylline, salbutamol and terbutaline in acute asthma. Indian J Chest Dis Allied Sci. 1984;26:155-158.

33. Siegel D, Sheppard D, Gelb A, Weinberg PF. Aminophylline increases the toxicity but not the efficacy of an inhaled beta-adrenergic agonist in the treatment of acute exacerbations of asthma. Am Rev Respir Dis. 1985;132:283-286.

34. Tribe AE, Wong RM, Robinson JS. A controlled trial of intravenous salbutamol and aminophylline in acute asthma. Med J Aust. 1976; 2:749-752.

35. Wrenn K, Slovis CM, Murphy F, Greenberg RS. Aminophylline therapy for acute bronchospastic disease in the emergency room. Ann Intern Med. 1991;115:241-247. 
36. Zainudin BM, Ismail O, Yusoff $\mathrm{K}$. Effect of adding aminophylline infusion to nebulised salbutamol in severe acute asthma. Thorax. 1994; 49:267-269.

37. Bien JP, Bloom MD, Evans RL, Specker B, O’Brien KP. Intravenous theophylline in pediatric status asthmaticus. A prospective, randomized, double-blind, placebo-controlled trial. Clin Pediatr Phila. 1995; $34: 475-481$.

38. Carter E, Cruz M, Chesrown S, Shieh G, Reilly K, Hendeles L. Efficacy of intravenously administered theophylline in children hospitalized with severe asthma. J Pediatr. 1993;122:470-476.

39. D'Avila RS, Piva JP, Marostica PJ, Amantea SL. Early administration of two intravenous bolus of aminophylline added to the standard treatment of children with acute asthma. Respir Med. 2008;102:156-161.

40. DiGiulio GA, Kercsmar CM, Krug SE, Alpert SE, Marx CM. Hospital treatment of asthma: lack of benefit from theophylline given in addition to nebulized albuterol and intravenously administered corticosteroid. J Pediatr. 1993;122:464-469.

41. Hambleton G, Stone MJ. Comparison of IV salbutamol with IV aminophylline in the treatment of severe, acute asthma in childhood. Arch Child. 1979;54:391-392.

42. Ibrahim SA, Elgurashi ED, Elkarim OA. Comparative study of intravenous aminophylline subcutaneous adrenaline and nebulised salbutamol in the treatment of acute asthma in children. Pediatr Rev Commun. 1993; 7:175-182.

43. Needleman JP, Kaifer MC, Nold JT, Shuster PE, Redding MM, Gladstein J. Theophylline does not shorten hospital stay for children admitted for asthma. Arch Pediatr Adolesc Med. 1995;149: 206-209.

44. Nuhoglu Y, Dai A, Barlan IB, Basaran MM. Efficacy of aminophylline in the treatment of acute asthma exacerbation in children. Ann Allergy Asthma Immunol. 1998;80:395-398.

45. Pierson WE, Bierman CW, Stamm SJ, Van Arsdel PP Jr. Double-blind trial of aminophylline in status asthmaticus. Pediatrics. 1971;48: 642-646.

46. Ream RS, Loftis LL, Albers GM, Becker BA, Lynch RE, Mink RB. Efficacy of IV theophylline in children with severe status asthmaticus. Chest. 2001;119:1480-1488.

47. Roberts G, Newsom D, Gomez K, et al. Intravenous salbutamol bolus compared with an aminophylline infusion in children with severe asthma: a randomised controlled trial. Thorax. 2003;58:306-310.

48. Singhi S, Grover S, Bansal A, Chopra K. Randomised comparison of intravenous magnesium sulphate, terbutaline and aminophylline for children with acute severe asthma. Acta Paediatr. 2014;103: 1301-1306.

49. Strauss RE, Wertheim DL, Bonagura VR, Valacer DJ. Aminophylline therapy does not improve outcome and increases adverse effects in children hospitalized with acute asthmatic exacerbations. Pediatrics. 1994; $93: 205-210$.

50. Tiwari A, Guglani V, Jat KR. Ketamine versus aminophylline for acute asthma in children: a randomized, controlled trial. Ann Thorac Med. 2016;11:283-288.

51. Vieira SE, Lotufo JP, Ejzenberg B, Okay Y. Efficacy of IV aminophylline as a supplemental therapy in moderate broncho-obstructive crisis in infants and preschool children. Pulm Pharmacol Ther. 2000;13: 189-194.
52. Wheeler DS, Jacobs BR, Kenreigh CA, Bean JA, Hutson TK, Brilli RJ. Theophylline versus terbutaline in treating critically ill children with status asthmaticus: a prospective, randomized, controlled trial. Pediatr Crit Care Med. 2005;6:142-147.

53. Yung M, South M. Randomised controlled trial of aminophylline for severe acute asthma. Arch Child. 1998;79:405-410.

54. Whig J, Puri S, Mahajan R, Chopra SC, Mittal N, Malhotra S. Placebo controlled evaluation of intravenous aminophylline in acute asthma. Lung India. 2001;19:97-100.

55. Williams SJ, Parrish RW, Seaton A. Comparison of intravenous aminophylline and salbutamol in severe asthma. Br Med J. 1975;4:685.

56. Rapid Respiratory Rate - A Sign of Asthma Problems [Internet] [cited May 29, 2017]. Available from: https://www.asthmasymptoms.org/ rapid-respiratory-rate.html. Accessed May 29, 2017.

57. Camargo CA, Rachelefsky G, Schatz M. Managing asthma exacerbations in the emergency department. Proc Am Thorac Soc. 2009; 6: 357-366.

58. Managing acute asthma in clinical settings [Internet]. Australian Asthma Handbook [cited Jun 2, 2017]. Available from: http://www. asthmahandbook.org.au/acute-asthma/clinical. Accessed June 2, 2017.

59. Pollart SM, Compton RM, Elward KS. Management of acute asthma exacerbations. Am Fam Physician. 2011;84:40-47.

60. Neame M, Aragon O, Fernandes RM, Sinha I. Salbutamol or aminophylline for acute severe asthma: how to choose which one, when and why? Arch Dis Child Educ Pract. 2015;100:215-222.

61. Dalabih AR, Bondi SA, Harris ZL, Saville BR, Wang W, Arnold DH. Aminophylline infusion for status asthmaticus in the pediatric critical care unit setting is independently associated with increased length of stay and time for symptom improvement. Pulm Pharmacol Ther. 2014;27:57-61.

62. Mitra AA, Bassler D, Watts K, Lasserson TJ, Ducharme FM. Intravenous aminophylline for acute severe asthma in children over two years receiving inhaled bronchodilators. Cochrane Database Syst. Rev. [Internet]. John Wiley \& Sons, Ltd; 2005. Available from: http:// onlinelibrary.wiley.com/doi/10.1002/14651858.CD001276.pub2/ abstract. Accessed June 2, 2017.

63. Travers AH, Jones AP, Camargo CA Jr, Milan SJ, Rowe BH. Intravenous beta2-agonists versus intravenous aminophylline for acute asthma. Cochrane Database Syst. Rev. [Internet]. John Wiley \& Sons, Ltd; 2012. Available from: http://onlinelibrary.wiley.com/doi/10.1002/14651858. CD010256/abstract. Accessed June 2, 2017.

64. Nair P, Milan SJ, Rowe BH. Addition of intravenous aminophylline to inhaled beta2-agonists in adults with acute asthma. Cochrane Database Syst. Rev. [Internet]. John Wiley \& Sons, Ltd; 2012. Available from: http://onlinelibrary.wiley.com/doi/10.1002/14651858.CD002742. pub2/abstract. Accessed June 2, 2017.

65. Morris I, Lyttle MD, O’Sullivan R, Sargant N, Doull IJM, Powell CVE. Which intravenous bronchodilators are being administered to children presenting with acute severe wheeze in the UK and Ireland? Thorax. 2015;70:88-91.
Drug Design, Development and Therapy

\section{Publish your work in this journal}

Drug Design, Development and Therapy is an international, peerreviewed open-access journal that spans the spectrum of drug design and development through to clinical applications. Clinical outcomes, patient safety, and programs for the development and effective, safe, and sustained use of medicines are the features of the journal, which

\section{Dovepress}

has also been accepted for indexing on PubMed Central. The manuscript management system is completely online and includes a very quick and fair peer-review system, which is all easy to use. Visit http://www.dovepress.com/testimonials.php to read real quotes from published authors. 